\title{
Optimum Biped Trajectory Planning for Humanoid Robot Navigation in Unseen Environment
}

\author{
Hanafiah Yussof ${ }^{1,2}$ and Masahiro Ohka ${ }^{1}$ \\ ${ }^{1}$ Graduate School of Information Science, Nagoya University \\ Japan \\ ${ }^{2}$ Faculty of Mechanical Engineering, Universiti Teknologi MARA \\ Malaysia
}

\section{Introduction}

The study on biped locomotion in humanoid robots has gained great interest since the last decades (Hirai et. al. 1998, Hirukawa et. al., 2004, Ishiguro, 2007). This interest are motivated from the high level of mobility, and the high number of degrees of freedom allow this kind of mobile robot adapt and move upon very unstructured sloped terrain. Eventually, it is more desirable to have robots of human build instead of modifying environment for robots (Khatib et. al, 1999). Therefore, a suitable navigation system is necessary to guide the robot's locomotion during real-time operation. In fundamental robot navigation studies, robot system is normally provided with a map or a specific geometrical guidance to complete its tasks (Okada et al., 2003, Liu et al., 2002). However during operation in uncertain environment such as in emergency sites like an earthquake site, or even in a room that the robots never been there before, which is eventually become the first experience for them, robots needs some intelligence to recognize and estimate the position and structure of objects around them. The most important is robot must localize its position within this environment and decide suitable action based on the environment conditions. To archives its target tasks, the robot required a highly reliable sensory devices for vision, scanning, and touching to recognize surrounding. These problems have become the main concern in our research that deals with humanoid robot for application in built-for-human environment.

Operation in unseen environment or areas where visual information is very limited is a new challenge in robot navigation. So far there was no much achievement to solve robot navigation in such environments. In previous research, we have proposed a contact interaction-based navigation strategy in a biped humanoid robot to operate in unseen environment (Hanafiah et al., 2008). In this chapter, we present analysis results of optimum biped trajectory planning for humanoid robot navigation to minimize possibility of collision during operation in unseen environment. In this analysis, we utilized 21-dof biped humanoid robot Bonten-Maru II. Our aim is to develop reliable walking locomotion in order 
to support the main tasks in the humanoid robot navigation system. Fig. 1 shows diagram of humanoid robot Bonten-Maru II and its configurations of dofs.

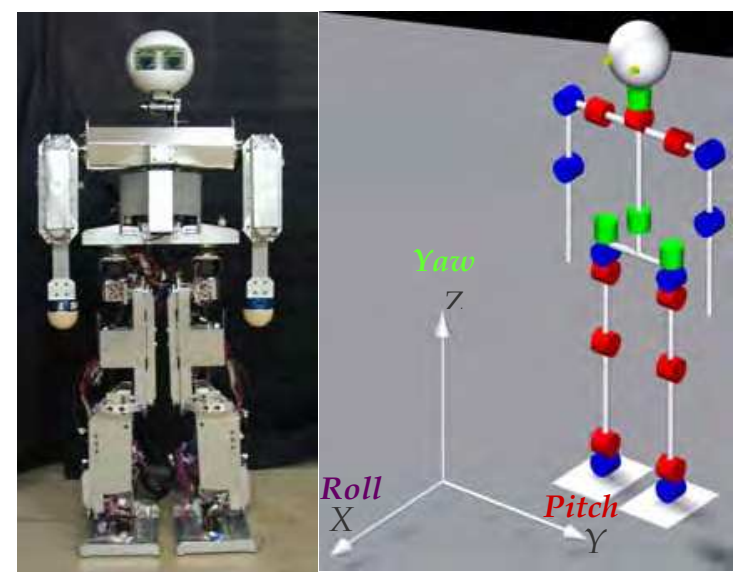

Fig. 1. Humanoid Robot Bonten-Maru II and its configuration of dofs.

It is inevitable that stable walking gait strategy is required to provide efficient and reliable locomotion for biped robots. In the biped locomotion towards application in unseen environment, we identified three basic motions: walk forward and backward directions, side-step to left and right, and yawing movement to change robot's orientation. In this chapter, at first we analyzed the joint trajectory generation in humanoid robot legs to define efficient gait pattern. We present kinematical solutions and optimum gait trajectory patterns for humanoid robot legs. Next, we performed analysis to define efficient walking gait locomotion by improvement of walking speed and travel distance without reducing reduction-ratio at joint-motor system. This is because sufficient reduction-ratio is required by the motor systems to supply high torque to the robot's manipulator during performing tasks such as object manipulation and obstacle avoidance. We also present optimum yawing motion strategy for humanoid robot to change its orientation within confined space. The analysis results were verified with simulation and real-time experiment with humanoid robot Bonten-Maru II.

Eventually, to safely and effectively navigate robots in unseen environment, the navigation system must feature reliable collision checking method to avoid collisions. In this chapter, we present analyses of collision checking using the robot arms to perform searching, touching and grasping motions in order to recognize its surrounding condition. The collision checking is performed in searching motion of the robot's arms that created a radius of detection area within the arm's reach. Based on the searching area coverage of the robot arms, we geometrically analyze the robot biped motions using Rapid-2D CAD software to identify the ideal collision free area. The collision free area is used to calculate maximum biped step-length when no object is detected. Consequently the robot control system created an absolute collision free area for the robot to generate optimum biped trajectories. In case of object is detected during searching motion, the robot arm will touch and grasp the object surface to define self-localization, and consequently optimum step-length is refined. 
Verification experiments were conducted using humanoid robot Bonten-Maru II to operate in a room with walls and obstacles was conducted. In this experiment, the robot visual sensors are not connected to the system. Therefore the robot locomotion can only rely on contact interaction of the arms that are equipped with force sensors.

\section{Short Survey on Humanoid Robot Navigation}

Operation in unseen environment or areas where visual information is very limited is a new challenge in robot navigation. So far there was no much achievement to solve robot navigation in such environments. In normal conditions, it is obvious that a navigation system that applies non-contact sensors such as vision sensors provides intensive information about the environment (Sagues \& Guerrero, 1999). However, robots cannot just rely on this type of sensing information to effectively work and cooperate with humans. For instance, in real applications the robots are likely to be required to operate in areas where vision information is very limited, such as in a dark room or during a rescue mission at an earthquake site (Diaz et. al., 2001). Moreover vision sensors have significant measurement accuracy problems resulting from technical problems such as low camera resolution and the dependence of stereo algorithms on specific image characteristics. Furthermore, the cameras are normally located at considerable distance from objects in the environment where operation takes place, resulting in approximate information of the environment.

In addition to the above, a laser range finder has also been applied in a robot navigation system (Thompson et. al., 2006). This sensor is capable of producing precise distance information and provides more accurate measurements compared with the vision sensor. However, it is impractical to embed this type of sensor with its vision analysis system in a walking robot system because of its size and weight (Okada et. al., 2003). A navigation system that applies contact-based sensors is capable of solving the above problems, particularly for a biped walking robot system (Hanafiah et. al., 2007). This type of sensor can accurately gauge the structure of the environment, thus making it suitable to support current navigation systems that utilize non-contact sensors. Furthermore, the system architecture is simpler and can easily be mounted on the walking robot body.

Eventually, to safely and effectively navigate robots in unseen environment, the navigation system must feature reliable collision checking method to avoid collisions. To date, in collision checking and prediction research, several methods such as vision based local floor map (Okada et al., 2003, Liu et al., 2002) and cylinder model (Guttmann et al., 2005) have been proposed for efficient collision checking and obstacle recognition in biped walking robot. In addition, Kuffner (Kuffner et al., 2002) have used fast distance determination method for self-collision detection and prevention for humanoid robots. This method is for convex polyhedra in order to conservatively guarantee that the given trajectory is free of self-collision. However, to effectively detect objects based on contact-based sensors, such methods are not suitable because they are mostly based on assumption of environment conditions acquired by non-contact sensors such as vision and laser range sensors.

Several achievements have been reported related with navigation in humanoid robots. Ogata have proposed human-robot collaboration based on quasi-symbolic expressions applying humanoid on static platform named Robovie (Ogata et al., 2005). This work combined non-contact and contact sensing approach in collaboration of human and robot during navigation tasks. This is the closest work with the approach used in this research. 
However Ogata use humanoid robot without leg. On the other hand, related with biped humanoid robot navigation, the most interesting work was presented by Stasse where visual 3D Simultaneous Localization and Mapping (SLAM) was used to navigate HRP-2 humanoid robot performing visual loop-closing motion (Stasse et al., 2006). In other achievements, Gutmann (Gutmann et al., 2005) have proposed real-time path planning for humanoid robot navigation. The work was evaluated using QRIO Sony's small humanoid robot equipped with stereo camera. Meanwhile, Seara have evaluated methodological aspects of a scheme for visually guided humanoid robot navigation using simulation (Seara et al., 2004). Next, Okada have proposed humanoid robot navigation system using vision based local floor map (Okada et al., 2003). Related with sensory-based biped walking, Ogura (Ogura et al., 2004) has proposed a sensory-based biped walking motion instruction strategy for humanoid robot using visual and auditory sensors to generate walking patterns according to human orders and to memorize various complete walking patterns. In previous research, we have proposed a contact interaction-based navigation strategy in a biped humanoid robot to operate in unseen environment (Hanafiah et. al., 2008). In this chapter, we present analysis results of optimum biped trajectory planning for humanoid robot navigation to minimize possibility of collision during operation in unseen environment.

\section{Simplification of Kinematics Solutions}

A reliable trajectory generation formulations will directly influence stabilization of robot motion especially during operation in unseen environment where the possibility of unstable biped walking due to ground condition and collision with unidentified objects are rather high if compared to operation in normal condition. In this chapter, at first we analyzed the joint trajectory generation in humanoid robot legs to define efficient gait pattern. We present kinematical solutions and optimum gait trajectory patterns for humanoid robot legs.

Eventually, formulations to generate optimum trajectory in articulated joints and manipulators are inevitable in any types of robots, especially for legged robot. Indeed, the most sophisticated forms of legged motion are that of biped gait locomotion. However calculation to solve kinematics problems to generate trajectory for robotic joints is a complicated and time-consuming study, especially when it involves a complex joint structure. Furthermore, computation of joint variables is also needed to compute the required joint torques for the actuators. In current research, to generate optimum robot trajectory, we simplified kinematics formulation to generate trajectory for each robot joint in order to reduce calculation time and increase reliability of robot arms and legs motions. This is necessary because during operation in unseen environment, robot will mainly rely on contact interaction using its arms. Consequently, an accurate and fast respond of robot's both legs are very important to maintain stability of its locomotion.

We implemented a simplified approach to solving inverse kinematics problems by classifying the robot's joints into several groups of joint coordinate frames at the robot's manipulator. To describe translation and rotational relationship between adjacent joint links, we employ a matrix method proposed by Denavit-Hartenberg (Denavit \& Hartenberg, 1955), which systematically establishes a coordinate system for each link of an articulated chain. Since this chapter focusing on biped trajectory, we present kinematical analysis of 6dofs leg in the humanoid robot Bonten-Maru II body. 


\subsection{Kinematical Solutions of 6-DOFs Leg}

Each of the legs has six dofs: three dofs (yaw, roll and pitch) at the hip joint, one dof (pitch) at the knee joint and two dofs (pitch and roll) at the ankle joint. In this research, we solve only inverse kinematics calculations for the robot leg. Figure 2 shows the structure and configuration of joints and links in the robot's leg. A reference coordinate is taken at the intersection point of the 3-dofs hip joint.

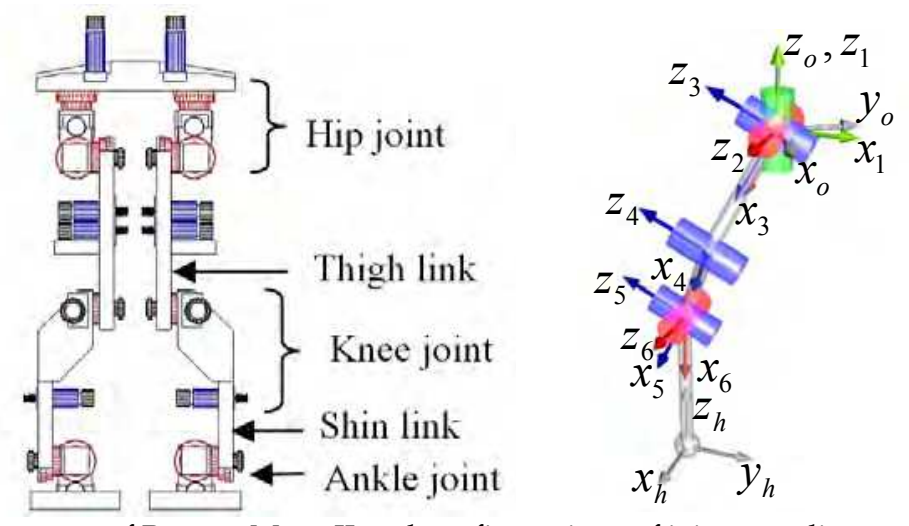

Fig. 2. Leg structure of Bonten-Maru II and configurations of joint coordinates.

\begin{tabular}{|c|c|c|c|c|}
\hline Link & $\theta_{\text {ileg }}$ & $d$ & $\alpha$ & $l$ \\
\hline 0 & $\theta_{1 \text { leg }}+90^{\circ}$ & 0 & 0 & 0 \\
\hline 1 & $\theta_{2 \text { leg }}-90^{\circ}$ & 0 & $90^{\circ}$ & 0 \\
\hline 2 & $\theta_{3 \text { leg }}$ & 0 & $90^{\circ}$ & 0 \\
\hline 3 & $\theta_{4 \text { leg }}$ & 0 & 0 & $l_{1}$ \\
\hline 4 & $\theta_{5 \text { leg }}$ & 0 & 0 & $l_{2}$ \\
\hline 5 & $\theta_{6 \text { leg }}$ & 0 & $-90^{\circ}$ & 0 \\
\hline 6 & 0 & 0 & 0 & $l_{3}$ \\
\hline
\end{tabular}

Table 1. Link parameters of the 6-dofs humanoid robot leg.

In solving calculations of inverse kinematics for the leg, the joint coordinates are divided into eight separate coordinate frames as listed bellow:

$\Sigma_{0}$ : Reference coordinate.

$\Sigma_{1}$ : Hip yaw coordinate.

$\Sigma_{2}$ : Hip roll coordinate.

$\Sigma_{3}$ : Hip pitch coordinate.

$\Sigma_{4}$ : Knee pitch coordinate.

$\Sigma_{5}$ : Ankle pitch coordinate.

$\Sigma_{6}:$ Ankle roll coordinate.

$\Sigma_{h}$ : Foot bottom-center coordinate. 
Figure 2 also shows a model of the robot leg that indicates the configurations and orientation of each set of joint coordinates. Here, link length for the thigh is $l_{1}$, while for the shin it is $l_{2}$. Link parameters for the leg are defined in Table 1. From the Denavit-Hartenberg convention mentioned above, definitions of the homogeneous transform matrix of the link parameters can be described as follows:

$$
{ }_{h}^{0} \mathbf{T}=\operatorname{Rot}\left(\mathrm{z}_{i}, \theta_{i}\right) \operatorname{Trans}\left(0,0, d_{i}\right) \operatorname{Trans}\left(l_{i}, 0,0\right) \operatorname{Rot}\left(\mathrm{x}_{i}, \alpha_{i}\right) .
$$

Here, variable factor $\theta_{i}$ is the joint angle between the $x_{i-1}$ and the $x_{i}$-axes measured about the $z_{i}$ axis; $d_{i}$ is the distance from the $x_{i-1}$ axis to the $x_{i}$ axis measured along the $z_{i}$ axis; $\alpha_{i}$ is the angle between the $z_{i}$ axis to the $z_{i-1}$ axis measured about the $x_{i-1}$ axis, and $l_{i}$ is the distance from the $z_{i}$ axis to the $z_{i-1}$ axis measured along the $x_{i-1}$ axis. Referring to Fig. 2, the transformation matrix at the bottom of the foot $\left({ }_{h}^{6} \mathbf{T}\right)$ is an independent link parameter because the coordinate direction is changeable. Here, to simplify the calculations, the ankle joint is positioned so that the bottom of the foot settles on the floor surface. The leg's orientation is fixed from the reference coordinate so that the third row of the rotation matrix at the leg's end becomes like equation (2).

$$
P_{z l e g}=\left[\begin{array}{lll}
0 & 0 & 1
\end{array}\right]^{T}
$$

Furthermore, the leg's links are classified into three groups to short-cut the calculations, where each group of links is calculated separately as follows:

i) From link 0 to link 1 (Reference coordinate to coordinate joint number 1).

ii) From link 1 to link 4 (Coordinate joint no. 2 to coordinate joint no. 4 ).

iii) From link 4 to link 6 (Coordinate joint no. 5 to coordinate at the bottom of the foot).

Basically, i) is to control leg rotation at the $z$-axis, ii) is to define the leg position, while iii) is to decide the leg's end-point orientation. A coordinate transformation matrix can be arranged as following.

$$
{ }_{h}^{0} \mathbf{T}={ }_{1}^{0} \mathbf{T}_{4}^{l} \mathbf{T}{ }_{h}^{4} \mathbf{T}=\left({ }_{h}^{0} \mathbf{T}\right)\left({ }_{2}^{l} \mathbf{T}_{3}^{2} \mathbf{T}{ }_{4}^{3} \mathbf{T}\right)\left({ }_{5}^{4} \mathbf{T}_{6}^{5} \mathbf{T}_{h}^{6} \mathbf{T}\right)
$$

Here, the coordinate transformation matrices for ${ }_{4}^{1} \mathbf{T}$ and ${ }_{h}^{4} \mathbf{T}$ can be defined as (4) and (5), respectively.

$$
{ }_{4}^{1} \mathbf{T}={ }_{2}^{1} \mathbf{T}_{3}^{2} \mathbf{T}_{4}^{3} \mathbf{T}
$$

$$
\begin{aligned}
& =\left[\begin{array}{cccc}
s_{2} c_{34} & -s_{2} s_{34} & -c_{2} & l_{1} s_{2} c_{3} \\
-s_{34} & -c_{34} & 0 & -l_{1} s_{3} \\
-c_{2} c_{34} & c_{2} s_{34} & -s_{2} & -l_{1} c_{2} c_{3} \\
0 & 0 & 0 & 1
\end{array}\right] \\
{ }_{h}^{4} \mathbf{T}={ }_{5}^{4} \mathbf{T}_{6}^{5} \mathbf{T}_{h}^{6} \mathbf{T} & =\left[\begin{array}{cccc}
c_{5} c_{6} & -c_{5} s_{6} & -s_{5} & l_{2}+l_{3} c_{5} c_{6} \\
s_{5} c_{6} & -s_{5} s_{6} & c_{5} & l_{3} s_{5} c_{6} \\
-s_{6} & -c_{6} & 0 & -l_{3} s_{6} \\
0 & 0 & 0 & 1
\end{array}\right]
\end{aligned}
$$

The coordinate transformation matrix for ${ }_{h}^{0} \mathbf{T}$, which describes the leg's end-point position and orientation, can be shown with the following equation. 


$$
{ }_{h}^{0} \mathbf{T}=\left[\begin{array}{cccc}
r_{11} & r_{12} & r_{13} & p_{x} \\
r_{21} & r_{22} & r_{23} & p_{y} \\
r_{31} & r_{32} & r_{33} & p_{z} \\
0 & 0 & 0 & 1
\end{array}\right]
$$

From equation (2), the following conditions were satisfied.

$$
r_{13}=r_{23}=r_{31}=r_{32}=0, r_{33}=1
$$

Hence, joint rotation angles $\theta_{1 \mathrm{leg}} \sim \theta_{6 \mathrm{leg}}$ can be defined by applying the above conditions. First, considering i), in order to provide rotation at the $z$-axis, only the hip joint needs to rotate in the yaw direction, specifically by defining $\theta_{1 \mathrm{leg}}$. As mentioned earlier, the bottom of the foot settles on the floor surface; therefore, the rotation matrix for the leg's end-point measured from the reference coordinate can be defined by the following equation.

${ }_{h}^{0} \mathbf{R}=\operatorname{Rot}\left(z, \theta_{l_{\text {leg }}}\right)$

$$
=\left[\begin{array}{ccc}
c \theta_{l_{\text {leg }}} & -s \theta_{l_{\text {leg }}} & 0 \\
s \theta_{l_{\text {leg }}} & c \theta_{l_{\text {leg }}} & 0 \\
0 & 0 & 1
\end{array}\right]=\left[\begin{array}{ccc}
r_{11} & r_{12} & 0 \\
r_{21} & r_{22} & 0 \\
0 & 0 & 1
\end{array}\right]
$$

Here, $\theta_{1 \text { leg }}$ can be defined as below.

$$
\theta_{1 \text { leg }}=\operatorname{atan} 2\left(r_{21}, r_{22}\right)
$$

Next, considering ii), from the obtained result of $\theta_{1 \operatorname{leg}},{ }_{h}^{0} \mathbf{T}$ is defined in (9).

$$
{ }_{h}^{0} \mathbf{T}=\left[\begin{array}{cccc}
-s_{1} & -c_{1} & 0 & P_{x \operatorname{leg}} \\
c_{1} & -s_{1} & 0 & P_{y_{\text {leg }}} \\
0 & 0 & 1 & P_{z \operatorname{leg}} \\
0 & 0 & 0 & 1
\end{array}\right]
$$

Here, from constrain orientation of the leg's end point, the position vector of joint 5 is defined as follows in (11), and its relative connection with the matrix is defined in (12). Next, equation (13) is defined relatively.

$$
\begin{aligned}
&{ }_{0} \mathbf{P}_{5}={ }_{4}^{0} \mathbf{T}^{4} \mathbf{P}_{5}= {\left[\begin{array}{lll}
P_{x \text { leg }} & P_{y_{\text {leg }}} & P_{z l \text { leg }}-l_{3}
\end{array}\right]^{T}, } \\
&{ }_{4} T^{4} \hat{P}_{5}={ }_{1}^{0} T^{-10} \hat{P}_{5} \\
& {\left[\begin{array}{cccc}
s_{2} c_{34} & -s_{2} s_{34} & -c_{2} & l_{1} s_{2} c_{3} \\
-s_{34} & -c_{34} & 0 & -l_{1} s_{3} \\
-c_{2} c_{34} & c_{2} s_{34} & -s_{2} & -l_{1} c_{2} c_{3} \\
0 & 0 & 0 & 1
\end{array}\right]\left[\begin{array}{c}
l_{2} \\
0 \\
0 \\
1
\end{array}\right]=\left[\begin{array}{cccc}
-s_{1} & c_{1} & 0 & 0 \\
-c_{1} & -s_{1} & 0 & 0 \\
0 & 0 & 1 & 0 \\
0 & 0 & 0 & 1
\end{array}\right]\left[\begin{array}{c}
p_{x} \\
p_{y} \\
p_{z}-l_{3} \\
1
\end{array}\right] }
\end{aligned}
$$

Therefore,

$$
\left[\begin{array}{l}
\hat{P}_{x \text { leg }} \\
\hat{P}_{y_{\text {leg }}} \\
\hat{P}_{z \text { leg }}
\end{array}\right]=\left[\begin{array}{l}
s_{2}\left(l_{1} c_{3}+l_{2} c_{34}\right) \\
-\left(l_{1} c_{3}+l_{2} s_{34}\right) \\
-c_{2}\left(l_{1} c_{3}+l_{2} c_{34}\right)
\end{array}\right] .
$$


To define joint angles $\theta_{2 \text { leg, }} \theta_{3 \text { leg, }} \theta_{4 \text { leg, }}$ equation (14) is used. Therefore, the rotation angles are defined as the following equations:

$$
\begin{gathered}
\theta_{4 \text { leg }}=\operatorname{atan} 2\left( \pm \sqrt{1-C^{2}}, C\right) \\
\theta_{3_{\text {leg }}}=\operatorname{atan} 2\left(\hat{p}_{x z \text { leg }}, \hat{p}_{y_{\text {leg }}}\right)+\operatorname{atan} 2\left(k_{1}, k_{2}\right) \\
\theta_{2_{\text {leg }}}=\operatorname{atan} 2\left(\hat{p}_{x \text { leg }}, \hat{p}_{z \text { leg }}\right) .
\end{gathered}
$$

Eventually, $C, \hat{p}_{x z \text { leg }}, k_{1}, k_{2}$ are defined as follows:

$$
\begin{gathered}
C=\frac{\hat{p}_{x \mathrm{leg}}{ }^{2}+\hat{p}_{y_{\text {leg }}{ }^{2}+\hat{p}_{z \mathrm{leg}}{ }^{2}-\left(l_{1}{ }^{2}+l_{2}^{2}\right)}^{2 l_{1} l_{2}}}{\hat{p}_{x z \text { leg }}=\sqrt{\hat{p}_{x \mathrm{leg}}{ }^{2}+\hat{p}_{z \mathrm{leg}}{ }^{2}}} \\
k_{1}=l_{1}+l_{2} c_{4}, k_{2}=-l_{2} s_{4}
\end{gathered}
$$

Finally, considering iii), joint angles $\theta_{5 \operatorname{leg}}$ and $\theta_{6} \operatorname{leg}$ are defined geometrically by the following equations:

$$
\begin{aligned}
& \theta_{5 \text { leg }}=-\theta_{3 \text { leg }}-\theta_{4 \text { leg }} \\
& \theta_{6 \text { leg }}=-\theta_{2 \text { leg }} .
\end{aligned}
$$

\subsection{Interpolation and Gait Trajectory Pattern}

A common way of making a robot's manipulator to move from start point to end point in a smooth, controlled fashion is to have each joint to move as specified by a smooth function of time $t$. Each joint starts and ends its motion at the same time, thus the robot's motion appears to be coordinated. In this research, we employ degree- 5 polynomial equations to solve interpolation from start point $P_{0}$ to end point $P_{f}$. Degree- 5 polynomial equations provides smoother gait trajectory compared to degree-3 polynomial equations which commonly used in robotic control. Velocity and acceleration at $P_{0}$ and $P_{f}$ are defined as zero; only the position factor is considered as a coefficient for performing interpolation.

$$
P(t)=a_{0}+a_{1} t+a_{2} t^{2}+a_{3} t^{3}+a_{4} t^{4}+a_{5} t^{5}
$$

Time factor at $P_{0}$ and $P_{f}$ are describe as $t_{0}=0$ and $t_{f}$, respectively. Here, boundary condition for each position, velocity and acceleration at $P_{0}$ and $P_{f}$ are shown at following equations. 


$$
\left.\begin{array}{l}
P(0)=a_{0}=P_{O} \\
\dot{P}(0)=a_{1}=\dot{P}_{O} \\
\dot{P}(0)=2 a_{2}=\ddot{P}_{o} \\
P\left(t_{f}\right)=a_{0}+a_{1} t_{f}+a_{2} t_{f}^{2}+a_{3} t_{f}^{3}+a_{4} t_{f}^{4}+a_{5} t_{f}^{5}=P_{f} \\
\dot{P}\left(t_{f}\right)=a_{1}+2 a_{2} t_{f}+3 a_{3} t_{f}^{2}+4 a_{4} t_{f}^{3}+5 a_{5} t_{f}^{4}=\dot{P}_{f} \\
\ddot{P}\left(t_{f}\right)=2 a_{2}+6 a_{3} t_{f}+12 a_{4} t_{f}^{2}+20 a_{5} t_{f}^{3}=\ddot{P}_{f}
\end{array}\right\}
$$

Here, coefficient $a_{i}(i=0,1,2,3,4,5)$ are defined by solving deviations of above equations. Results of the deviations are shown at below equations.

$$
\begin{aligned}
& a_{0}=y_{0} \\
& a_{1}=\dot{y}_{0} \\
& a_{2}=\frac{1}{2} \ddot{y}_{0} \\
& a_{3}=\frac{1}{2 t_{f}^{3}}\left\{20\left(y_{f}-y_{0}\right)-\left(8 \dot{y}_{f}+12 \dot{y}_{o}\right) t_{f}+\left(\ddot{y}_{f}-3 \ddot{y}_{o}\right) t_{f}^{2}\right\} \\
& a_{4}=\frac{1}{2 t_{f}^{4}}\left\{-30\left(y_{f}-y_{0}\right)+\left(14 \dot{y}_{f}+16 \dot{y}_{o}\right) t_{f}-\left(2 \ddot{y}_{f}-3 \ddot{y}_{o}\right) t_{f}^{2}\right\} \\
& a_{5}=\frac{1}{2 t_{f}^{5}}\left\{12\left(y_{f}-y_{0}\right)-6\left(\dot{y}_{f}+\dot{y}_{o}\right) t_{f}+\left(\ddot{y}_{f}-\ddot{y}_{o}\right) t_{f}^{2}\right\}
\end{aligned}
$$

As mentioned before, velocity and acceleration at $P_{0}$ and $P_{f}$ were considered as zero, as shown in (26).

$$
\dot{P}(0)=\stackrel{\bullet}{P}(0)=\stackrel{\bullet}{P}\left(t_{f}\right)=\ddot{P}\left(t_{f}\right)=0 .
$$

Generation of motion trajectories from points $P_{0}$ to $P_{f}$ only considered the position factor. Therefore, by given only positions data at $P_{0}$ and $P_{f}$, respectively described as $y_{0}$ and $y_{f}$, coefficients $a_{i}(i=0,1,2,3,4,5)$ were solved as below.

$$
\left.\begin{array}{l}
a_{0}=y_{0} \\
a_{1}=0 \\
a_{2}=0 \\
a_{3}=\frac{10}{t_{f}^{3}}\left(y_{f}-y_{0}\right) \\
a_{4}=-\frac{15}{t_{f}^{4}}\left(y_{f}-y_{0}\right) \\
a_{5}=\frac{6}{t_{f}^{5}}\left(y_{f}-y_{0}\right)
\end{array}\right\}
$$

Finally, degree- 5 polynomial function is defined as following equation.

$$
y(t)=y_{o}+10\left(y_{f}-y_{o}\right) u^{3}-15\left(y_{f}-y_{o}\right) u^{4}+6\left(y_{f}-y_{o}\right) u^{5}
$$


Where,

$$
u=\frac{t}{t_{f}}=\frac{\text { current time }}{\text { motion time }} .
$$

These formulations provide smooth and controlled motion trajectory to the robot's manipulators during performing tasks in the proposed navigation system. Consequently, to perform a smooth and reliable gait, it is necessary to define step-length and foot-height during transferring one leg in one step walk. The step-length is a parameter value that can be adjusted and fixed in the control system. On the other hand, the foot-height is defined by applying ellipse formulation, like shown in gait trajectory pattern at Fig. 3. In case of walking forward and backward, the foot height at $z$-axis is defined in (30). Meanwhile during side steps, the foot height is defined in (31).

$$
\begin{aligned}
& z=b\left(1-\frac{x^{2}}{a^{2}}\right)^{\frac{1}{2}}-h \\
& z=b\left(1-\frac{y^{2}}{a^{2}}\right)^{\frac{1}{2}}-h
\end{aligned}
$$

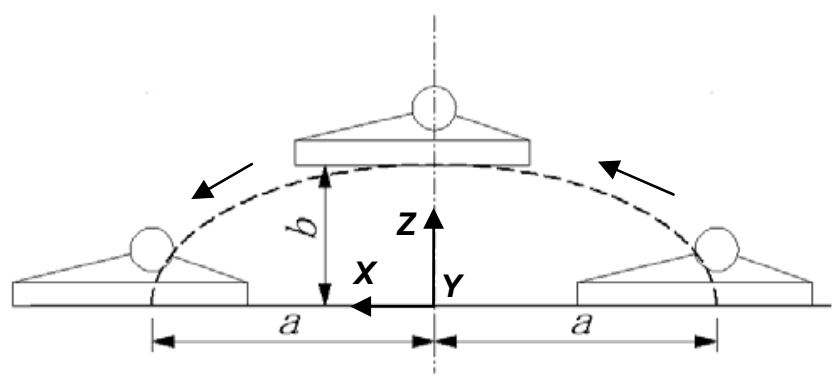

Fig. 3. Gait trajectory pattern of robot leg.

Here, $h$ is hip-joint height from the ground. In real-time operation, biped locomotion is performed by giving the leg's end point position to the robot control system so that joint angle at each joint can be calculated by inverse kinematics formulations. Consequently the joint rotation speed and biped trajectory pattern are controlled by formulations of interpolation. By applying these formulations, each gait motion is performed in smooth and controlled trajectory.

\section{Analysis of Biped Trajectory Locomotion}

It is inevitable that stable walking gait strategy is required to provide efficient and reliable locomotion for biped robots. In the biped locomotion towards application in unseen environment, we identified three basic motions: walk forward and backward directions, side-step to left and right, and yawing movement to change robot's orientation. In this section, we performed analysis to define efficient walking gait locomotion by improvement of walking speed and travel distance without reducing reduction-ratio at joint-motor system. 
This is because sufficient reduction-ratio is required by the motor systems to supply high torque to the robot's manipulator during performing tasks such as object exploration and obstacle avoidance. We also present optimum yawing motion strategy for humanoid robot to change its orientation within confined space.

\subsection{Human Inspired Biped Walking Characteristics}

Human locomotion stands out among other forms of biped locomotion chiefly in terms of the dynamic systems point of view. This is due to the fact that during a significant part of the human walking motion, the moving body is not in static equilibrium. The ability for humans to perform biped locomotion is greatly influenced by their learning ability (Dillmann, 2004, Salter et al., 2006). Apparently humans cannot walk when they are born but they can walk without thinking that they are walking as years pass by. However, robots are not good at learning. They are what they are programmed to do. In order to perform biped locomotion in robots, we must at first understand human's walking pattern and then develop theoretical strategy to perform the correct joint trajectories synthesis on the articulated chained manipulators at the robot's legs.

Figure 4 shows divisions of the gait cycle in human which focusing on right leg. Each gait cycle is divided into two periods, stance and swing. These often are called gait phase. Stance is the term used to designate the entire period during which the foot is on the ground. Both start and end of stance involve a period of bilateral foot contact with the floor (double stance), while the middle portion of stance has one foot contact. Stance begins with initial contact of heel strike, also known as initial double stance which begins the gait circle. It is the time both feet are on the floor after initial contact. The word swing applies to the time the foot is in the air for limb advancement. Swing begins as the foot is lifted from the floor. It was reported that the gross normal distribution of the floor contact periods is $60 \%$ for stance and $40 \%$ for swing (Perry, 1992). However, the precise duration of these gait cycle intervals varies with the person's walking velocity. The duration of both gait periods (stance and swing) shows an inverse relationship to walking speed. That is, both total stance and swing times are shortened as gait velocity increases. The change in stance and swing times becomes progressively greater as speed slows.

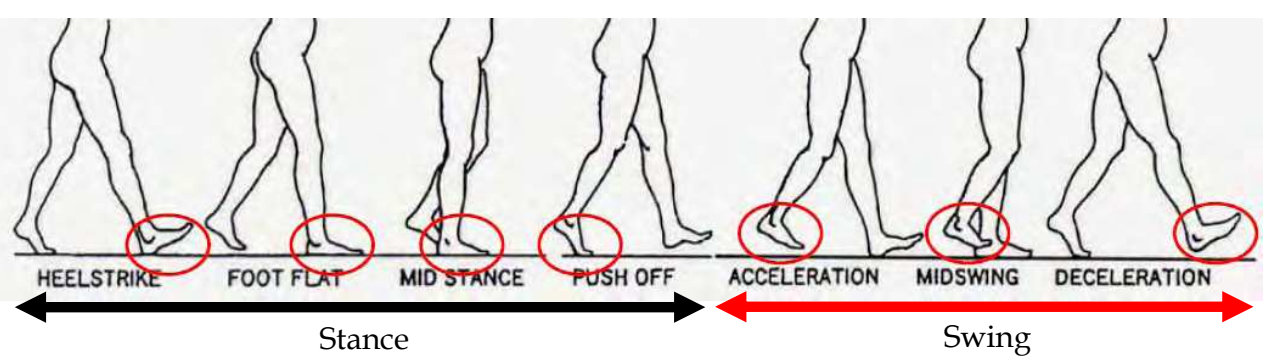

Fig. 4. Walking gait cycle in human. 


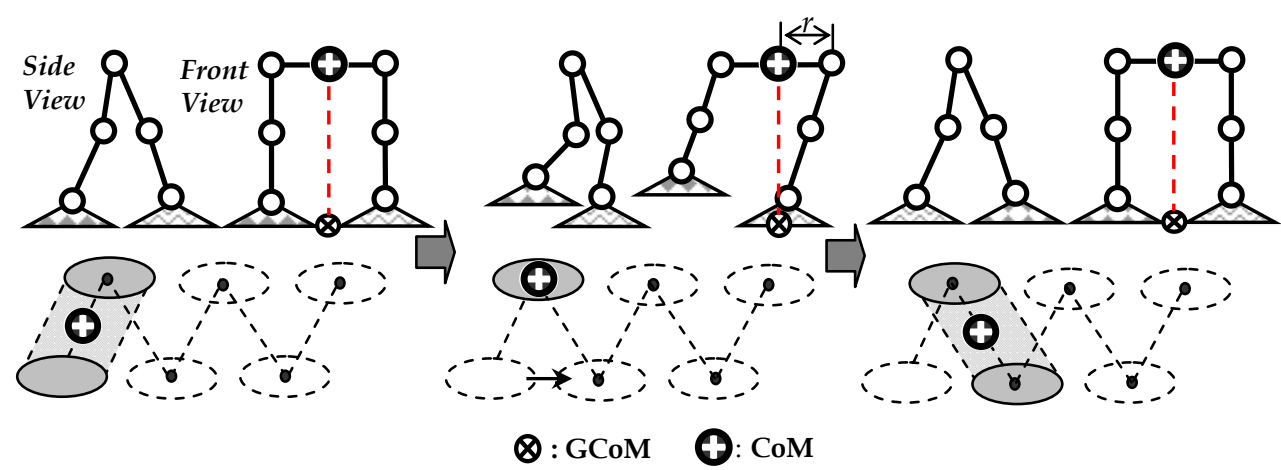

Fig. 5. Static walking model for biped robot in one cycle.

In contrast, for a biped robot two different situations arise in sequence during the walking motion: the statically stable double-support phase in which the whole structure of the robot is supported on both feet simultaneously, and the statically unstable single-support phase when only one foot is in contact with the ground, while the other foot is being transferred from back to front. Biped walking robot can be classified by its gait. There are two major research areas in biped walking robot: the static gait and dynamic gait. To describe gait motion in walking robots, it is easier to at first look at the static walking pattern point of view. In static walking pattern, two terms are normally used: Center of Mass (CoM) and Ground Projection of Center of Mass (GCoM). It is understood that to realize a stable gait motion, Center of Mass (CoM) and Ground Projection of Center of Mass (GCoM) must be in a straight line where the GCoM must always be within the foot sole area, as shown in Fig. 5 . If GCoM is outside of the foot sole area, the robot will lose balance and fall down.

Notice that when swinging one leg, the waist moves to be on top of another leg in order to shift CoM position so that the CoM is centered with the GCoM. These movements bring together the whole robot trunk to left and right simultaneously. Therefore, to safely navigate the biped locomotion in a humanoid robot, it is necessary to consider the trunk movement of the robot body. In this study, the trunk movement is considered as a parameter value $r$, as shown in Fig. 5, which is taken as the distance from waist-joint to hip-joint. Eventually, this kind of walking pattern delays the walking speed. Moreover, joint structure design in robots does not permit flexible movement like that of human being. Indeed, one motor only can rotate in one direction. Even by reducing reduction-ratio can increase the motor rotation, it will eventually reduce the torque output which is not desirable for real-time operation.

Therefore, instead of stabilization issue that have been presented in many research, analysis to increase walking speed is necessary in biped locomotion so that the robots can move faster without reducing the reduction-ratio at the motor system, which will jeopardize their ability to perform tasks. Furthermore, not many works have been reported regarding analysis of biped walking speed.

\subsection{Analysis of Biped Walking Speed}

We have identified that five main tasks need to be solved in the contact-based navigation system for biped robots: searching, self-localization, correction, obstacle avoidance, and object handling. On top of these tasks, walking locomotion is the basic motion that supports 
the tasks. It is therefore an efficient biped locomotion strategy in walking motion is required in the navigation system. For the sake of navigating a biped humanoid robot, the objective is to generate efficient gait during performing tasks and maintain in stable condition until the tasks are completed.

The efficiency in biped robots is normally related with how fast and how easy the tasks can be completed. In fact, a method to control sufficient walking speed in conjunction with the biped gait trajectory is inevitably important. This is because in real-time application, the robots are likely to be required to walk faster or slower according to situation that occurred during the operation. It is therefore we need to identify parameters to control walking speed in biped locomotion. Previously, several studies have been reported related with walking speed of biped robot. For example Chevallereau \& Aoustin (Chevallereau \& Aoustin, 2001) have studied optimal reference trajectory for walking and running of a biped robot.

Furthermore, Yamaguchi (Yamaguchi et al., 1993) have been using the ZMP as a criterion to distinguish the stability of walking for a biped walking robot which has a trunk. The authors introduce a control method of dynamic biped walking for a biped walking robot to compensate for the three-axis (pitch, roll and yaw-axis) moment on an arbitrary planned ZMP by trunk motion. The authors developed a biped walking robot and performed a walking experiment with the robot using the control method. The result was a fast dynamic biped walking at the walking speed of $0.54 \mathrm{~s} / \mathrm{step}$ with a $0.3 \mathrm{~m}$ step on a flat floor. This walking speed is about $50 \%$ faster than that with the robot which compensates for only the two-axis (pitch and roll-axis) moment by trunk motion. Meanwhile, control system that stabilizes running biped robot HRP-2LR has been proposed by Kajita (Kajita et al., 2005). The robot uses prescribed running pattern calculated by resolved momentum control, and a running controller stabilizes the system against disturbance.

In this research, we focus in developing efficient walking locomotion by improving the walking gait velocity and travel distance. This analysis employs the humanoid robot Bonten-Maru II as an analysis platform. Eventually, it is easy to control the walking speed by reducing or increasing the reduction-ratio at the robot joint-motor system. However, in real-time operation it is desirable to have a stable and high reduction-ratio value in order to provide high torque output to the robot's manipulator during performing tasks, such as during object manipulation, avoiding obstacle, etc. Therefore the reduction-ratio is required to remain always at fixed and high value.

\subsubsection{Selection of Parameters}

The main consideration in navigating a biped humanoid robot is to generate the robot's efficient gait during performing tasks and maintain it in a stable condition until the tasks are completed. The efficiency in biped robots is normally related with how fast and how easy the tasks can be completed. In this research, to increase walking speed without changing the reduction-ratio, we considered three parameters to control the walking speed in biped robot locomotion:

1) Step length; $s$

2) hip-joint height from the ground; $h$

3) Duty-ratio; $d$

Figure 6 shows initial orientation of Bonten-Maru II during motion mode which also indicate the step length and hip-joint height of the robot. The step-length is the distance between ankle-joints of a support leg and a swing leg when both of them are settled on the 
ground during walking motion. The hip-joint height is the distance between intersection point of hip-joint roll and pitch to the ground in walking position. Meanwhile, duty-ratio for biped robot mechanism is described as time ratio of one foot touches the ground when another foot swing to transfer the leg in one cycle of walking motion.

In biped gait motion, two steps are equal to one cycle (refer Fig. 5). Figure 6 also shows link dimension of the Bonten-Maru II body and structure of the leg. The link parameters at the legs are used in calculations to define hip-joint height and maximum step length by geometrical analysis. Link parameters of the legs were calculated geometrically to define relation between step-length and hip-joint height. From the geometrical analysis, relation between the step-length and the hip-joint height is defined in Table 2.

At joint-motor system of Bonten-Maru II, maximum no-load rotation for the DC servomotor at each joint is 7220 [rpm]. This rotation is reduced by pulley and harmonic drive-reduction system to $1 / 333$, in order to produce high torque output during performing tasks. We considered that the robot required high torque to perform tasks; therefore we do not change the reduction-ratio, which is 333:1. Eventually, these specifications produced maximum joint angular velocity at 130 [deg/s]. However, for safety reason, the joint angular velocity at the motor was reduced to 117 [deg/s].

The step time can be adjusted in the robot control system easily. However, if the step time is too small in order to increase walking speed, the robot motion becomes unstable. Moreover, the maximum step length performed becomes limited. In current condition, the step time for Bonten-Maru II to complete one cycle of walking is fixed between 7 10 second at maximum step length $75[\mathrm{~mm}]$. The duty-ratio $d$ is increased gradually from 0.7 to 0.85 .
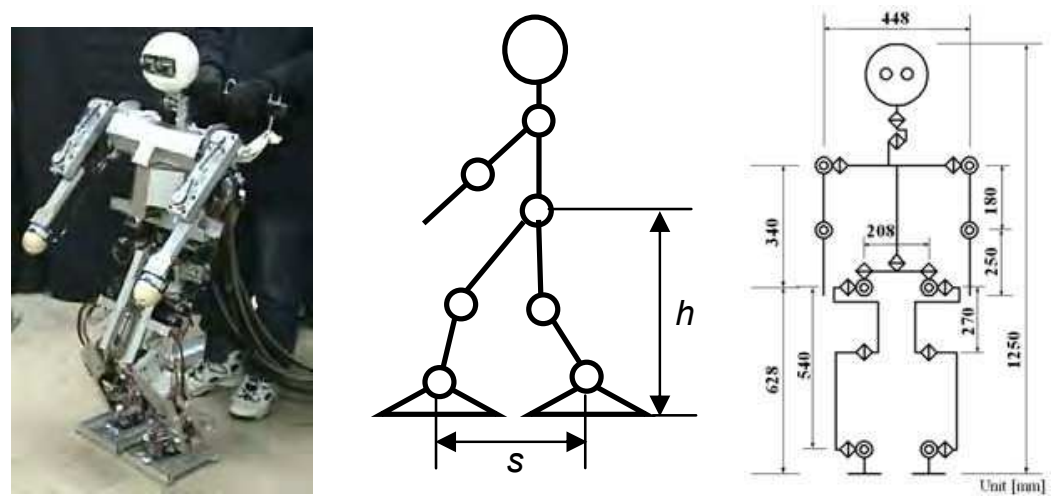

Fig. 6. Orientation of Bonten-Maru II to perform motion, parameters of hip-height $h$ and step length $s$, and diagram of link dimensions.

\begin{tabular}{|c|c|c|}
\hline Hip-joint height [mm] & $\begin{array}{c}\text { Max. step length in 1 } \\
\text { step }[\mathrm{mm}]\end{array}$ & $\begin{array}{c}\text { Max. step length in 1 } \\
\text { cycle [mm] }\end{array}$ \\
\hline$h_{1}=468$ & 350 & 700 \\
\hline$h_{2}=518$ & 300 & 600 \\
\hline$h_{3}=568$ & 200 & 400 \\
\hline
\end{tabular}

Table 2. Relationship of step length against hip-joint height at Bonten-Maru II. 


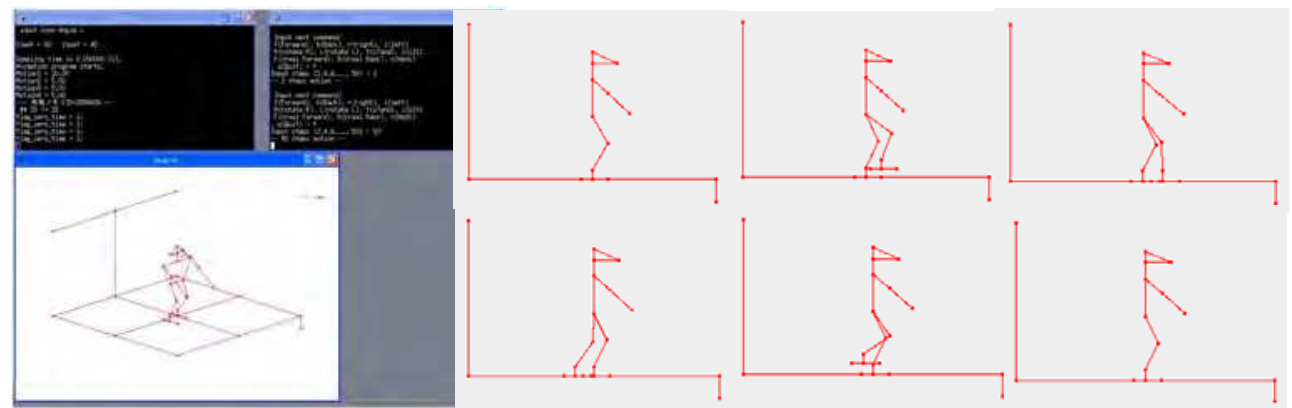

Fig. 7. Simulation by animation presents robot's trajectory in biped walking motion.

\subsubsection{Simulation Analysis}

A simulation analysis of the robot walking velocity using animation that applies GnuPlot was performed based on parameters condition explained at previous section. The time for one circle of walking gait is initially fixed at 10 second. Figure 7 displays the animation screen of the robot's trajectory, which features a robot animation performing walking motion. Each joint's rotation angles are saved and analyzed in a graph structure. Based on the joint angle, angular velocity of each joint was calculated.

For example, Fig. 8 shows joint angle data for right leg joints when performing 10 steps walk at condition: $h=518[\mathrm{~mm}], s=100[\mathrm{~mm}]$ and $d=0.7$. From the angle data, angular velocity for each joint was calculated and presented in Fig. 9. The first and last gait shows acceleration and deceleration of the gait velocity. The three steps in the middle show maximum angular velocity of the legs joint. Basically, in biped robot the maximum walking gait velocity is calculated from maximum joint angular velocity data by defining minimum step time for one gait. Eventually, by applying the same parameter, even if time for one step is initially different; the final joint angle obtained by the robot is same. Hence, in this analysis we can obtain the minimum step time in one step from the maximum joint angular velocity data that the initial step time was 10 seconds. Basically, the minimum gait time in one step is satisfying following equation:

$$
t_{\min }<\frac{v_{\theta \max } \times 10}{V_{\theta \max }} .
$$

Here, $V_{\theta \max }$ is the maximum joint angular velocity at the motor, $t_{\min }$ is minimum time for one step, and $v_{\theta \max }$ is maximum joint angular velocity in each gait. Finally, the maximum walking gait velocity $w_{\max }$ is defined by dividing length $s$ with minimum step time $t_{\min }$ in each gait, as shown in following equation.

$$
w_{\min }=\frac{s}{t_{\min }}
$$




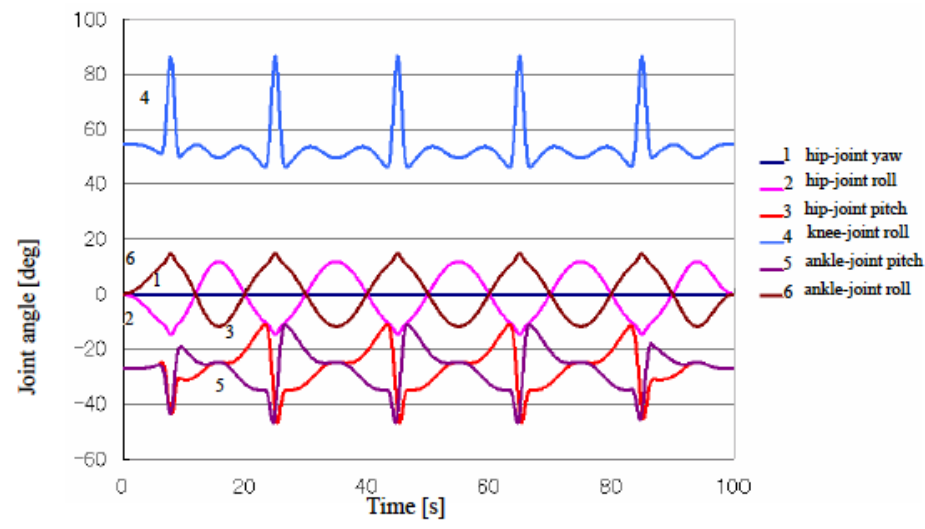

Fig. 8. Graph of joint rotation angle at right leg.

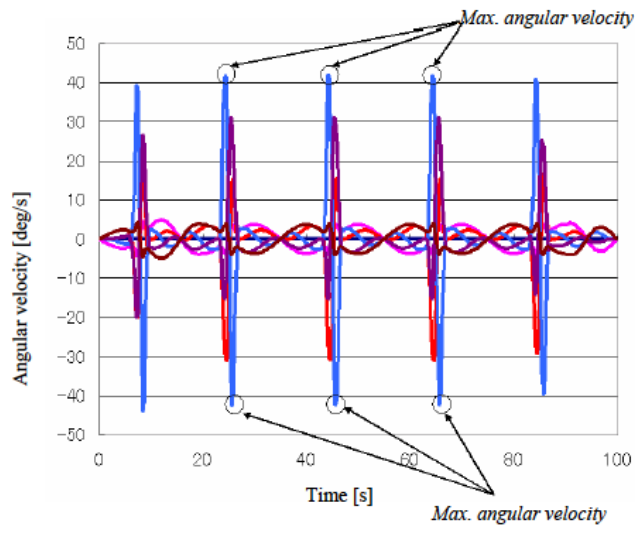

Fig. 9. Graph of angular velocity of joint rotation at right leg. 


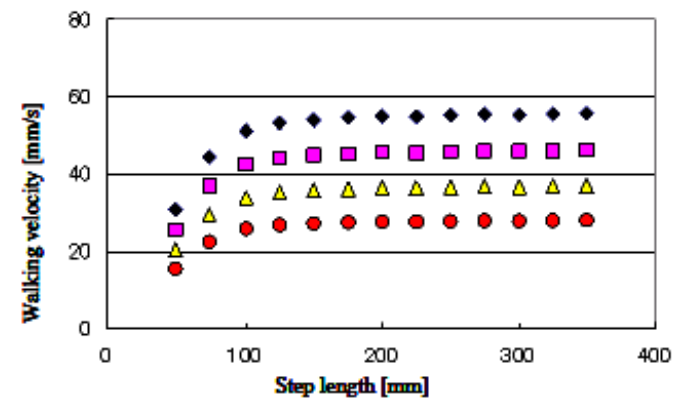

(a) Hip-joint height $468[\mathrm{~mm}]$

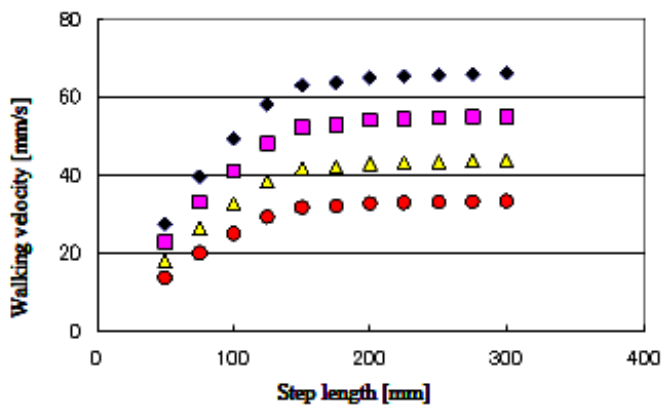

(b) Hip joint height 518 [mm]

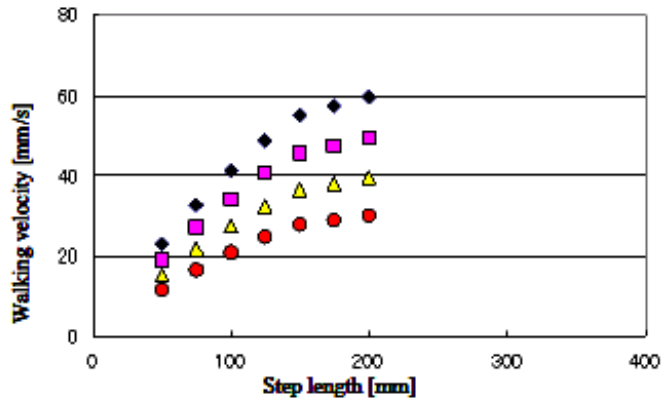

(c) Hip-joint height 568 [mm]

Duty-ratio: $: 0.7, \square: 0.75, \triangle 0.8, \quad 0.85$

Fig. 10. Analysis results of maximum walking velocity at each gait.

\subsubsection{Simulation Results}

Simulation results of walking gait velocity at each parameters value are compiled in graphs as shown in Fig. 10(a), (b) and (c). According to these graphs, from the relation of walking velocity and step length, the walking velocity was maintain nearly at constant value when it reached certain step length. Moreover, in relation of step length and hip-joint height, the higher hip-joint position is providing wider step length to perform better walking distance. 
At this point, lower duty-ratio shows the best results in relation of the hip-joint height and the step length for higher walking gait velocity, as shown in Fig. 10(b), where the low dutyratio shows high walking velocity in relationship between the hip-joint-height and the steplength. It means by shorten the time for the support leg touching the ground will urge swing leg to increase its speed to complete one walking cycle, thus increase the walking velocity. At the same time, by choosing suitable step-length and hip-joint-height parameters, travel distance in each step can be improved. This analysis results revealed that it is possible to control biped walking speed without reducing the reduction-ratio at the joint-motor system. From the simulation results, we can conclude that lower duty-ratio in suitable hip-joint height comparatively provided higher walking gait velocity. For Bonten-Maru II, the maximum walking gait velocity was improved from $30[\mathrm{~mm} / \mathrm{s}]$ to $66[\mathrm{~mm} / \mathrm{s}]$, which is about two times better than current walking velocity. At this time the hip-joint height is 518 $[\mathrm{mm}]$ and the time for one step is 4.5 seconds.

\subsubsection{Experiment with Bonten-Maru II}

We conduct experiments with the biped humanoid robot Bonten-Maru II. The parameter values that revealed the best result in simulation were applied, in comparison with current walking condition. Figures 11 and 12 respectively show photograph of the actual robot's walking motion in each experiment, which also indicate the parameter values applied. Travel distance was measured during the experiments. The experimental results show that by applying the best parameters value obtained in the simulation results, the walking speed was improved. At the same time, the travel distance is longer about three times compared with current condition.

This result reveals that the travel distance was improved in conjunction with the improvement of walking speed in the biped humanoid robot. The robot performed biped walking in smooth and stable condition. The experiments utilizing real biped humanoid robot based on simulation results shows that the robot's travel distance during walking was improved about three times better than current walking condition. This analysis proved that it is possible to improve walking speed in stable biped locomotion without reducing the reduction-ratio. This analysis results contributes to reliable biped locomotion during performing tasks in the humanoid robot navigation system.

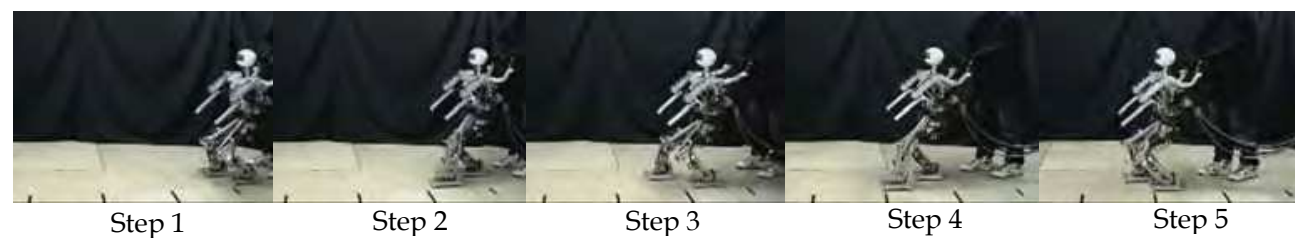

Fig. 11. Humanoid robot performs biped walking applying the best parameters value from simulation results: $h=518[\mathrm{~mm}], s=200[\mathrm{~mm}]$ and $d=0.7$, time per step $4.5 \mathrm{sec}$. 


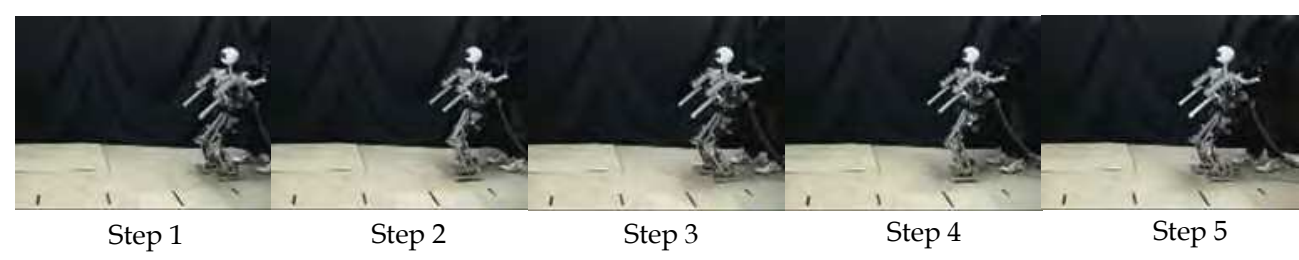

Fig. 12. Humanoid robot performs biped walking applying current parameters value: $h=568$ $[\mathrm{mm}], s=75[\mathrm{~mm}]$ and $d=0.8$, time per step $2.5 \mathrm{sec}$.

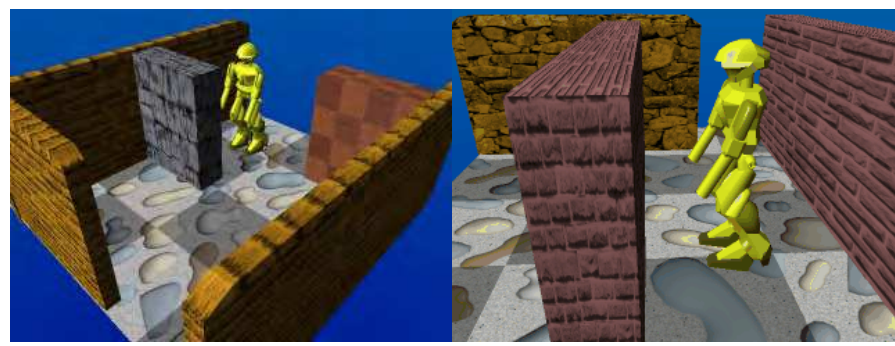

Fig. 13. Humanoid robot operates in confined space.

\subsection{Analysis of Yawing Motion}

To effectively operate in unseen environment, it is necessary for humanoid robot to have skills to operate in limited space, such as in a small room or a tunnel as shown in Fig. 13. In navigation within confined space, it is critical for biped robots to change their orientation within a very limited space in order to change locomotion direction. Motivated by this situation, we analyzed humanoid robot with its rigid body structure to define biped locomotion strategy to perform yawing movement towards operation in confined spaces. In this study, we identified two types of yawing movement to change the robot orientation at this situation. The first movement, so called pattern 1, is when the robot changed its orientation to the front-left or front-right area. The second movement called pattern 2 is when the robot turn to the back-left or back-right area. Geometrical analyses of these two yawing patterns for left side movements are illustrated in Fig. 14. Here, $m, n, \delta$ and $\psi$ are dimensional results from calculations to define each leg's position which taking hip-jointheight, length of the leg's links and maximum step length as parameter values. The $x y$-axes gives reference coordinates before rotation, while the $x^{\prime} y^{\prime}$-axes gives coordinates after rotation is completed. In order to change the robot's orientation, rotation of hip-joint yaw is the key-point, as shown in Fig. 15.

By solving inverse kinematics calculation to define joint rotation angle $\theta_{1 \text { leg }}$ as presented in previous section, yawing rotation of the leg was performed. The robot rotation angle was decided from 0 degree to 90 degree for both patterns. Meanwhile target position of leg's end-point at $X-Y$ axes plane defined by interpolation formulations distinguishing leg's movements of the pattern 1 and pattern 2. Motion planning for pattern 1 and pattern 2 are shown in Fig. 16. For example, in the case of rotating to left side, at first the left leg's hipjoint yaw will rotate counterclockwise direction to $\theta_{1}$ (see Fig. 15). At the same time, the left leg follow along an ellipse trajectory in regard to $z$-axis direction to move the leg one step. 
This stepping motion is performed by given the leg's end point position to the robot's control system so that the joint angles of $\theta_{1 l e g} \sim \theta_{6 l e g}$ could be solved by inverse kinematics.

The left leg position is defined by interpolation of the leg end point from its initial position with respect to the $x y$-axes position at a certain calculated distance (see Fig. 14). At this time the right leg acts as the support axis. Then, the robot corrects its orientation by changing the support axis to the left leg, while the left leg hip-joint yaw reverses the rotation to clockwise direction to complete the motion. These yawing movements minimize the rotation space of the robot, which consequently minimize the possibility of collision with objects around the robot. Furthermore, it is easier in control whereby only one leg performing rotation, while the other leg functioned as support axis. The rotation angle at hip-joint yaw also permits wide rotation range.

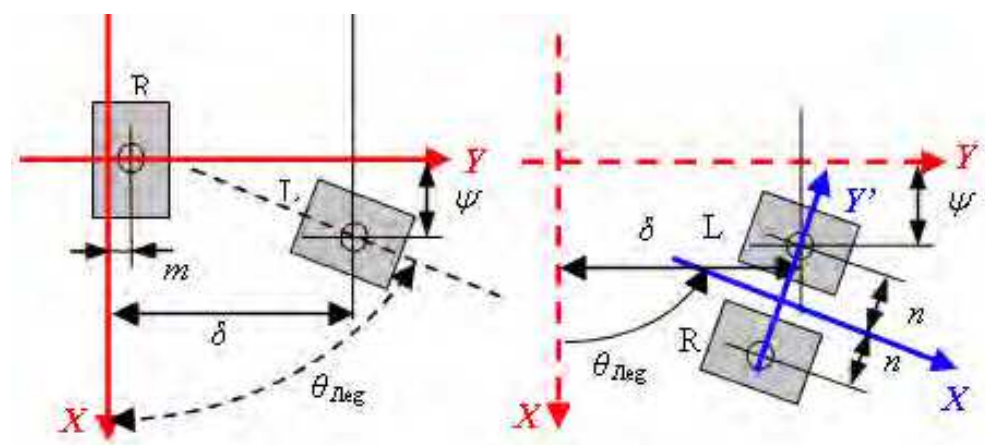

(i) Pattern 1
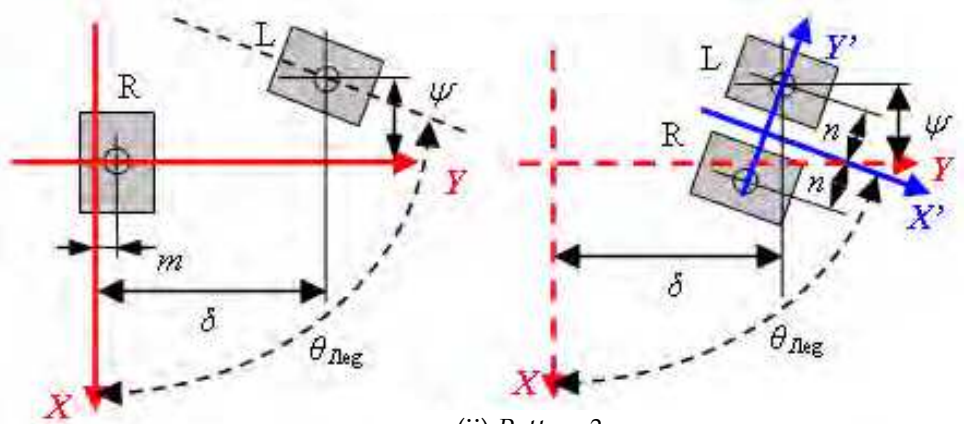

(ii) Pattern 2

Fig. 14. Geometrical analysis of the robot's foot-bottom position during yawing movements of pattern 1 and pattern 2 . 

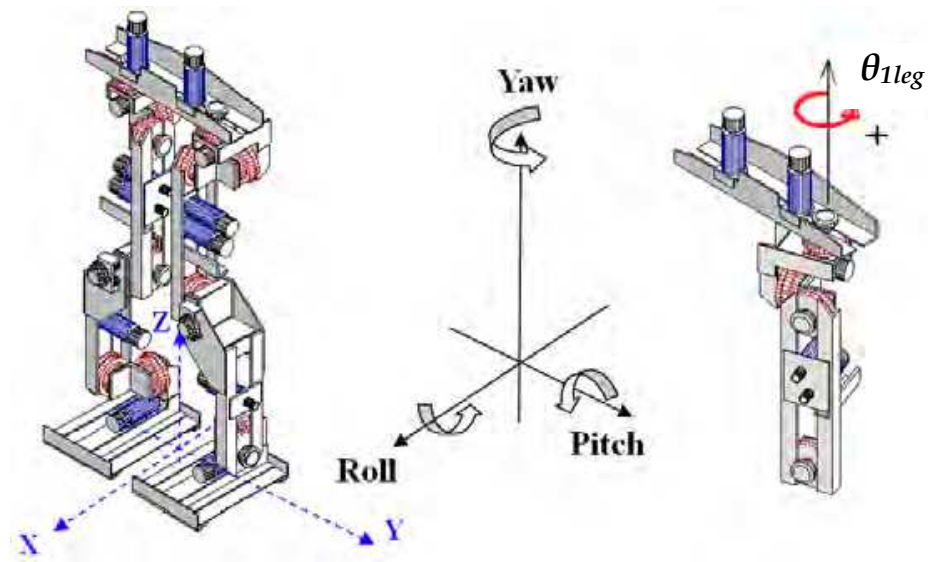

Fig. 15. Leg structure and rotation of hip-joint yaw.

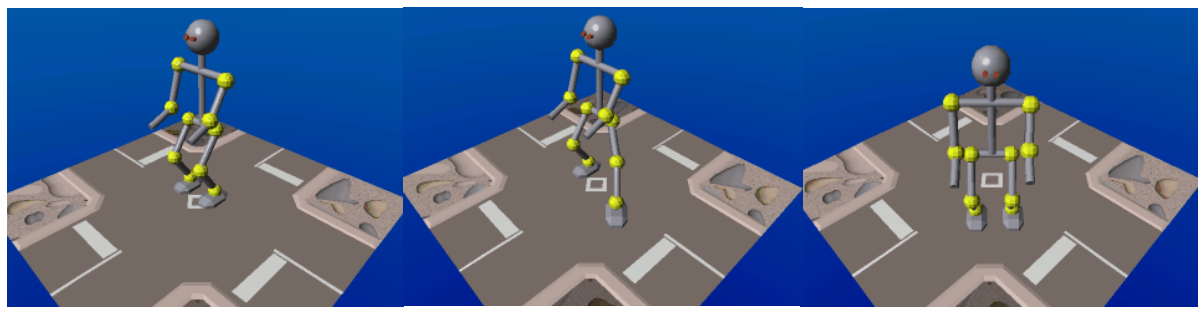

Pattern 1

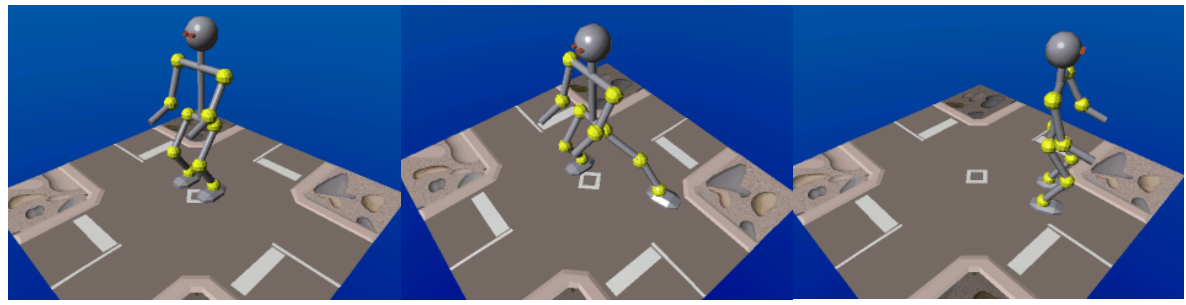

Pattern 2

Fig. 16. Motion planning of yawing motions in biped humanoid robot.

\section{Analysis of Collision Avoidance}

Eventually, to safely and effectively navigate robots in unseen environment, the navigation system must feature reliable collision checking method to avoid collisions. The purpose of analyzing collision checking in this research is to define collision free area based on the arm's detection area in searching tasks. The aim is to avoid collision with external objects so that the robot can perform walking motions safely in the navigation tasks. In conjunction with this collision free area, a suitable step-size of the robot's foot during walking motion is defined. We assumed that no objects were detected in the searching process, so that we can 
calculate maximum size of the collision free area. Consequently, when object is detected in searching process, the robot control system can recalculate the collision free area automatically based on the object's distance from robot.

Since the proposed navigation system in this research only depends on contact interaction of both arms to realize environment conditions, calculation of arm's links parameters and detection radius area in correlation with biped walk characteristics are important. Therefore, the collision checking method proposed in this research is considered the link parameters of the robot's arms, and trunk movement during biped walk motions as parameter values. The collision free area is used to calculate maximum biped step-length when no object is detected. Consequently the robot control system created an absolute collision free area for the robot to generate optimum biped trajectories. In case of object is detected during searching motion, the robot arm will touch and grasp the object surface to define selflocalization, and consequently optimum step-length is refined.

In this chapter, we present analyses of collision checking using the robot arms to perform searching, touching and grasping motions in order to recognize its surrounding condition. The collision checking is performed in searching motion of the robot's arms that created a radius of detection area within the arm's reach. Based on the searching area coverage of the robot arms, we geometrically analyze the robot biped motions using Rapid-2D CAD software to identify the ideal collision free area. The inputs are humanoid robot BontenMaru II link dimensions and joint rotation range as shown in Fig. 17. The outputs are radiuses of both arm rotations indicates detection area which taking centre of rotation at shoulder and elbow joints and absolute collisions free trajectory area of the robot legs.

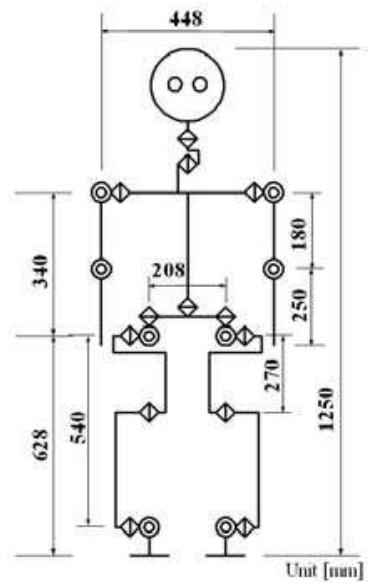

\begin{tabular}{|l|c|}
\hline \multicolumn{1}{|c|}{ Joint (Axis) } & Range of rotation angle [deg.] \\
\hline Right shoulder (roll) & $-135 \sim 30$ \\
\hline Left shoulder (roll) & $-30 \sim 135$ \\
\hline Elbow (roll) & $-90 \sim 90$ \\
\hline Hip (yaw) & $-90 \sim 90$ \\
\hline Right hip (roll) & $-90 \sim 25$ \\
\hline Left hip (roll) & $-25 \sim 90$ \\
\hline Hip (pitch) & $-130 \sim 45$ \\
\hline Knee (pitch) & $-20 \sim 150$ \\
\hline Ankle (pitch) & $-90 \sim 60$ \\
\hline Right ankle (roll) & $-90 \sim 20$ \\
\hline Left ankle (roll) & $-20 \sim 90$ \\
\hline
\end{tabular}

Fig. 17. Humanoid robot Bonten-Maru II link dimensions and joint rotation range.

\subsection{Methodology}

The purpose of analyzing collision checking in this research is to define collision free area based on the arm's detection area in searching tasks, and calculate suitable step-length of the robot leg in biped walking motions. The strategy will help humanoid robot to reduce possibility of collisions with external objects, at the same time help the robot control system to define optimum biped gait trajectories in the proposed navigation system. 
In the analysis, at first we assumed that no objects were detected in the searching process, so that we can define the maximum size of collision free area and consequently calculate the maximum step-length of the robot leg during walking to forward, backward and side directions.. Meanwhile, when object is detected in searching process, the robot can measure the collision free area based on the acquired object's distance and the specified maximum step-length. Accordingly, optimum step-length can be defined automatically in the robot control system.

Since the proposed navigation system in this research only depends on contact interaction of both arms to realize environment conditions, we must consider link dimensions of the robot's arm and the leg as parameter values. Furthermore, consideration of optimum arm's motions during searching tasks and its correlation with biped walk characteristics is also important. For Bonten-Maru II, we utilized parameters of link dimensions at the arms and legs as shown in Fig. 17. Meanwhile, motion of arms during searching tasks is classified in two patterns: First is the motion where rotation is centered at elbow joint, which is during searching object at the robot front area, and second is the motion where rotation is centered at shoulder joint, which is during searching object at robot side area.

\subsection{Condition I: When No Object Detected}

Initially, we perform analysis assuming that no object is detected in searching area so that we can identify the optimum collision free area and maximum step-length. The proposed arm rotation as explained in previous section created circular shape areas as shown in Fig. 18 , which radius $r_{1}$ is the arm's upper link, and $r_{2}$ is additional of lower and upper arm links parameters. These radiuses are actually replicating the arm's end-effector position during searching tasks. Based on the searching area coverage of the robot arms, we geometrically analyze the robot biped motions using Rapid-2D CAD software to identify the ideal collision free area. The relation of hip-joint height from ground $h$ and step-length $S$ in biped walking robot, as shown in Fig. 6, is considered in the analysis. In our research utilizing humanoid robot Bonten-Maru II, the robot hip-joint height during walking motion was fixed at $h=518[\mathrm{~mm}]$. Meanwhile, upper and lower link parameters of the arms are $L_{1}=180$ $[\mathrm{mm}], L_{2}=250[\mathrm{~mm}]$, respectively.

Figure 19 shows analysis result using Rapid-2D CAD software of the robot performing searching motion and biped walking to various directions. In this figure, at first the robot starts the motion by searching process. Then it walk to forward, backward and side-step directions, turn to left and right directions at 45 degree, and turn back to right and left directions at 70 degree. We considered the body trunk movements, foot sole positions, and the arms and legs rotation range at all walking directions to define the collision free area. Finally, we defined a rectangular shaped collision free area as shown in the Fig. 6. This figure also indicated radius of collision checking area replicating the arms end-effector position, and also foot sole area.

Eventually, as shown in Fig. 20, we can still see some areas within the rectangular shaped collision free trajectory area are out of the coverage of the collision checking radius. However, these areas are actually mostly covered when the robot start moving, for example as shown in Fig. 20 (right) where the robot performed side step motion to right-side direction. Indeed, it called absolute collision free trajectory area because the area is covered almost all walking directions where the robot can safely perform biped walking trajectories to satisfy tasks in the proposed navigation system. From the analysis result, we defined the 
maximum step-length for Bonten-Maru II when no object was detected: $75 \mathrm{~mm} / \mathrm{step}$ for walking to forward and backward, $100 \mathrm{~mm} / \mathrm{step}$ for walking to side-step, and $75 \mathrm{~mm} / \mathrm{step}$ for walking to tangential directions.
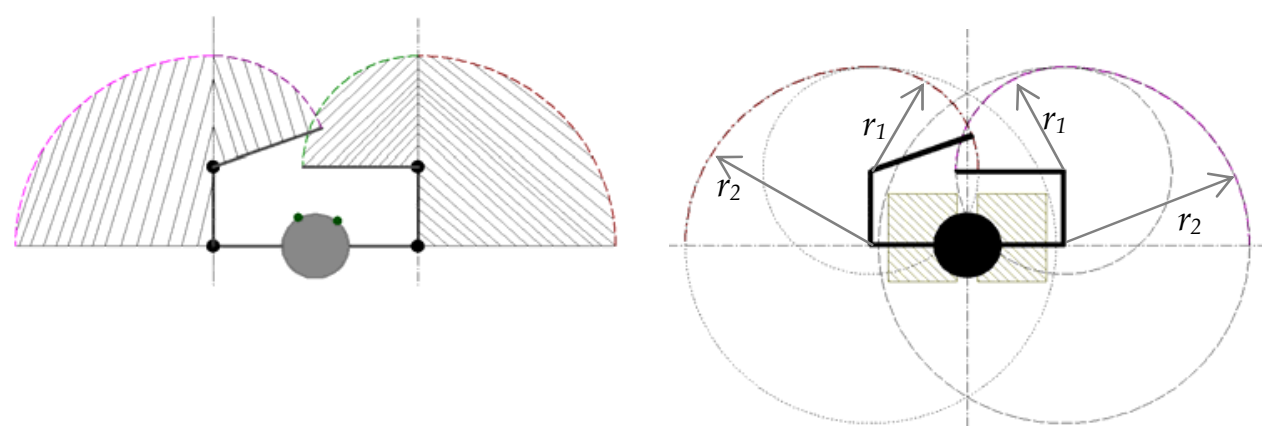

Fig. 18. (Left) Actual searching coverage area of both arms. (Right) Radiuses of both arm rotations indicates detection area taking centre of rotation at shoulder and elbow joints.

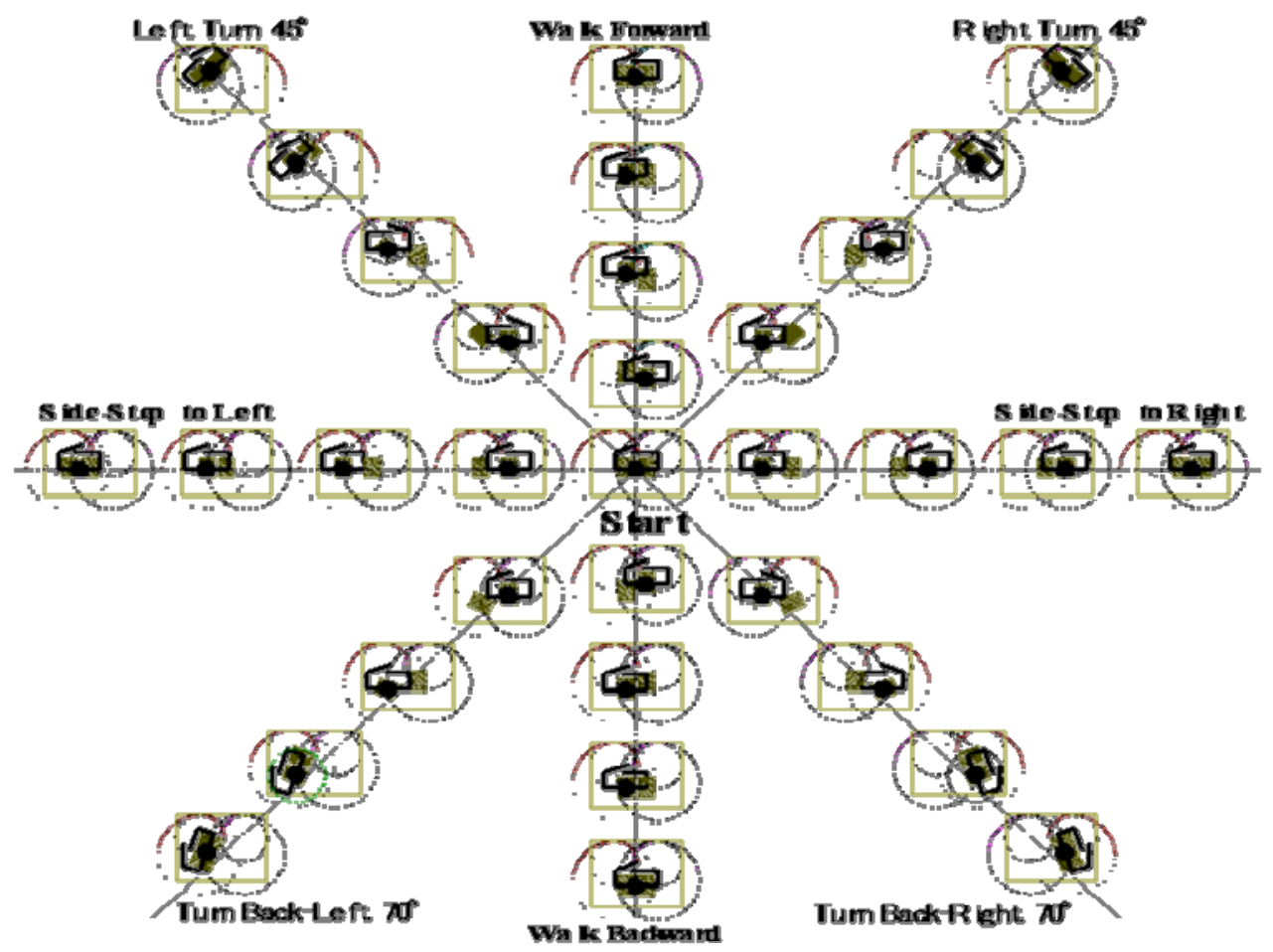

Fig. 19. Analysis of collision checking for humanoid robot Bonten-Maru II in walking forward, backward, side to left and right, and tangential directions using Rapid-2D software. 

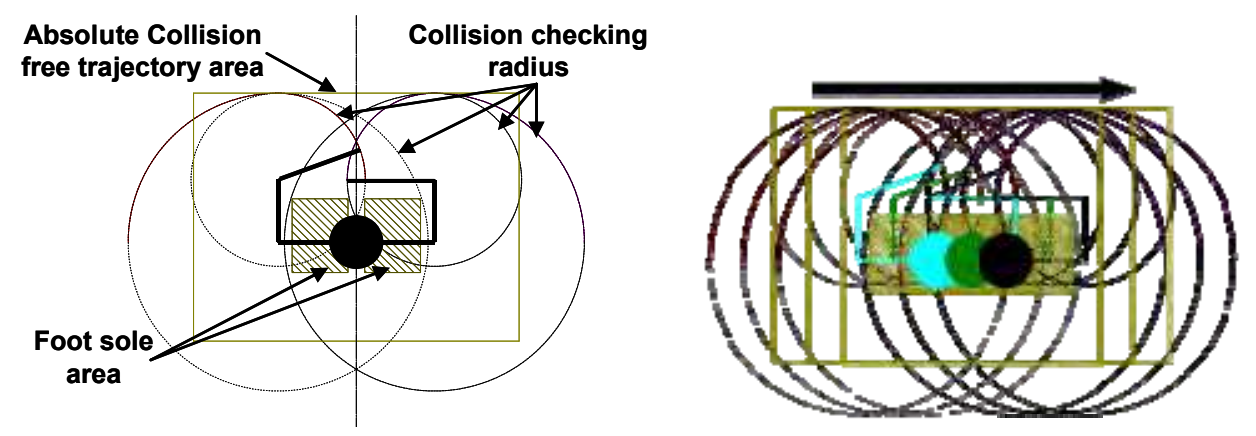

Fig. 20. (Left) Absolute collisions free trajectory area and collision checking parameters. (Right) Collision checking areas in walking side-step to right direction.

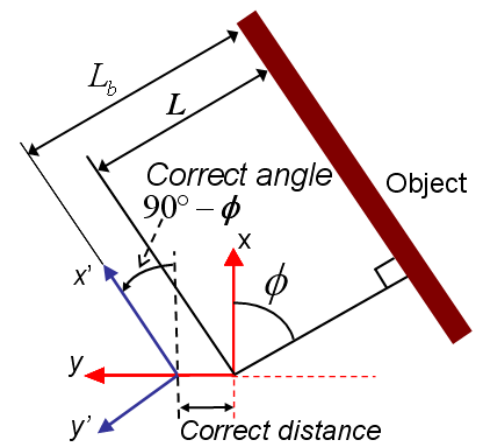

Fig. 21. Robot orientation after grasping process.

\subsection{Condition II: When Object Detected}

In situation where object is detected during searching tasks, the robot arm will grasp the object surface and define self-localization. The control system will measure the object orientation and then consequently calculate the robot distance and angle. Figure 21 shows the geometrical analysis of the robot position and angle after the grasping process. During grasping process, the arm's end-effector position data were defined. The position data are calculated with the least-square method to result for a linear equation, as shown in (34) where $a$ is the slope of the line and $b$ is the intersection at $y$-axis. A straight line from the reference coordinates origin that is perpendicular with (34), which describes the shortest distance from robot to wall, is defined in (35). Consequently, the intersection coordinate at the $x$ - $y$ axes plane is defined in (36). Here, grasping angle $\phi$ is an angle from the $x$-axis of the robot reference coordinates to the perpendicular line of (35). Finally, the distance of the robot to object $L$ and grasping angle $\phi$ are shown in (37) and (38), respectively.

$$
\begin{gathered}
y=a x+b \\
y=-\frac{1}{a} x
\end{gathered}
$$




$$
\begin{aligned}
\left(C_{x}, C_{y}\right) & =\left(-\frac{a b}{a^{2}+1}, \frac{b}{a^{2}+1}\right) \\
L & =\frac{b}{\sqrt{a^{2}+1}} \\
\phi & =\tan ^{-1}\left(-\frac{1}{a}\right)
\end{aligned}
$$

For collision checking, the size of collision free area is refined automatically in the robot control system based on parameter value of shortest distance $L$. Consequently, a parameter value $L_{b}$ which considered safety distance between the robot to the object during walking locomotion is defined based on link parameters of the arm. Accordingly, the step-length $s$ of the robot during biped walking is recalculate using shortest distance $L$ and grasping angle $\phi$ as indicated in (38).
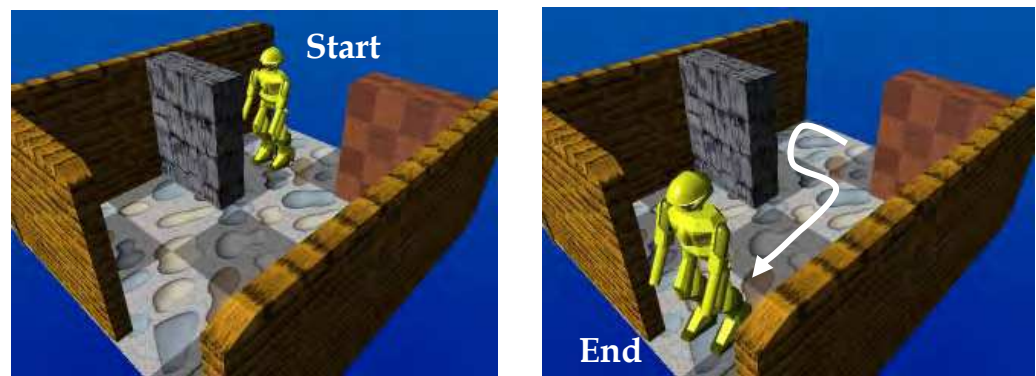

Fig. 22. Layout of Humanoid robot operates in small room with wall and obstacle.

\section{Experiment}

Verification experiments using humanoid robot Bonten-Maru II to operate in a room with walls and obstacles was conducted. The walls and obstacles were arranged as shown in Fig. 22. In this experiment, the robot visual sensors are not connected to the system. Therefore the robot locomotion can only rely on contact interaction of the arms that are equipped with force sensors. A human operator acts as the motion instructor and controls the robot motions. The operator has prior knowledge of the room arrangement but does not know the exact starting position of the robot.

\subsection{Motion Planning}

Observation of human behavior during operation in a dark room shows that at first the human subject seems to search and identify the nearest object to recognize his position in the room. Inspired by this scenario, the humanoid robot starts the navigation process by searching for the nearest object to define self-localization so that it can recognize its position and orientation in the environment where the operation takes place. Continuously the robot generates suitable locomotion based on contact information; as described by the proposed algorithm explained in the previous section.

Lastly, the navigation of a walking robot requires accurate collision avoidance parameters. 
This ensures the safety of robot when it performs locomotion in the navigation tasks. As mentioned in section 4, the robot trunk movement parameter $r$ is one of the parameters which need to be considered. The arm detection area which is equal to the length of arm $L_{t}$ also needs to be considered as a parameter. The robot travel distance (step length and quantity of steps) in walking motions is controlled based on these parameter values. For forward and backward walking motion, travel distance must be less than the value of $L_{t}$. Whereas for the side-stepping motion and tangential walking during the correction process, the travel distance is fixed to be less than parameter value of $L_{t}+r$.

In order to explain motion planning in this experiment, we simulate room with walls and obstacles to overview the path planning of the proposed method. Figure 23 shows top view of the robot orientation while it performs locomotion in the simulation room. The robot starts the locomotion by searching for nearest wall using right arm. If no wall is detected, robot will walk side-step to its right direction and repeats the searching process until the arm detects a wall. When the wall is detected, the robot's arm will grasp the wall surface to obtain the wall's orientation. Base on the obtained wall orientation, the robot control system could recognize its position and orientation against the wall.

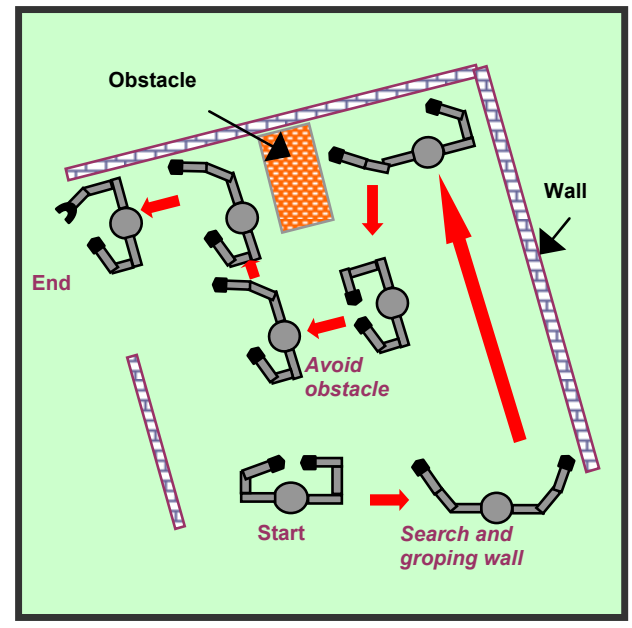

Fig. 23. Motion planning in navigation experiment.

Next, the robot performs locomotion to correct its orientation, at first by correcting its position. After correction of position, the robot's left arm will check the existence of any obstacles at the targeted correction of angle area. If no obstacle is detected, the robot will continue correcting its orientation by changing its direction angle. If the obstacle is detected, the robot will perform trajectories to avoid the obstacle. The robot will repeat the whole process again until it reaches to the desired target point. In this research, the robot control system is intelligently classified each process so that the robot could effectively perform suitable locomotion according to the surrounding conditions. 


\subsection{Motion Algorithm}

In the proposed humanoid robot navigation strategy, we designed a motion algorithm consisting of searching and detecting object, self-localization by grasping, correction of locomotion direction, and obstacle avoidance (Hanafiah et al., 2008). Figure 24 shows the flowchart of the motion algorithm. The algorithm comprises formulations of kinematics, interpolation of manipulator's end-effector, and force-position control in order to generate trajectory for each robotic joint.

First the robot searches the nearest object using both arms. When no object was detected, the robot walked side-step to its right direction. In this navigation algorithm, navigation priority was given to right-side direction so that optimum motions could be achieved during navigation tasks. Then the robot repeats again the search and detection tasks. When the object was detected, the robot grasped its surface to define self-localization. Self-localization here means calculation results of distance and orientation of each humanoid robot's joints towards the object. Then the robot checked for any obstacle existence in the correction area using its left arm. When no obstacle was detected, the robot corrected its locomotion direction by performing yawing motions with both legs. Then the robot repeats again the searching process. The robot continued its locomotion while touching and avoiding objects in its path. When it was necessary, the robot changed its direction by turning right or left.

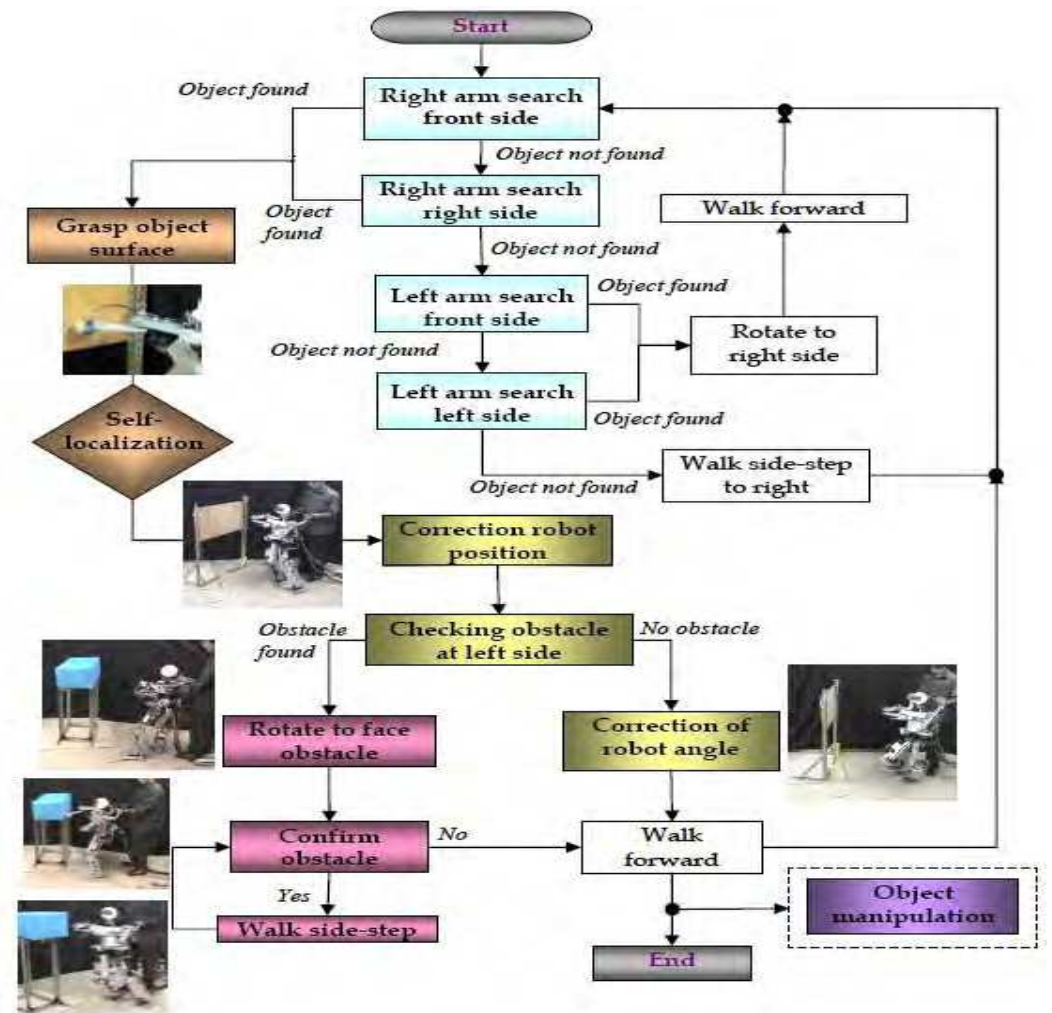

Fig. 24. Layout of motion algorithm in contact interaction-based navigation. 


\subsection{Experiment Result}

Since the robot's vision sensors were not connected to the system, the robot locomotion had to rely on the contact interaction of the arms equipped with force sensors. The proposed navigation algorithm was installed in the robot control system.

Figure 25 shows sequential photographs of actual robot locomotion during the experiment. At first the robot was searching for the nearest object using both arms. When the object was detected, the robot grasped the object surface to define self-localization. Then the robot corrected its locomotion direction after checking for obstacle in the correction area. The robot continued its locomotion while touching and avoiding objects along its way. When it was necessary, the robot changed its direction by turning to right and left. Finally, the robot managed to complete the navigation tasks safely and reached the target end point. Experimental results revealed good performance of the robot to realize collision free area and generate optimum biped walking.

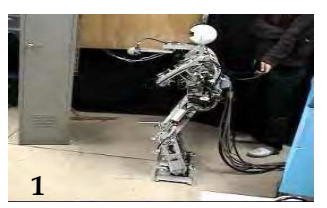

Search front and right side

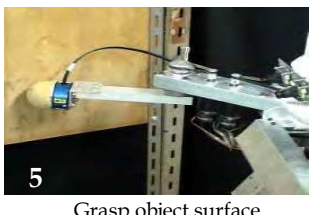

Grasp object surface
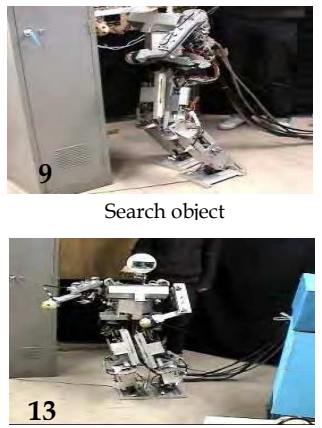

Search front and right side

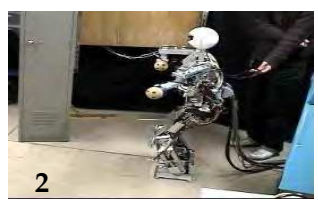

Search front and left side

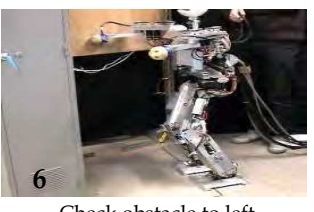

Check obstacle to left

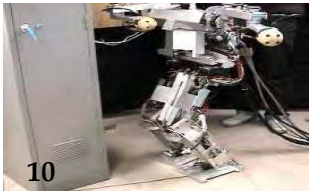

Check obstacle

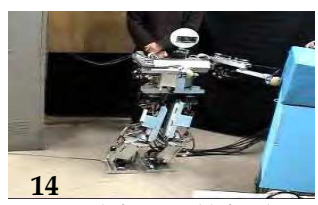

Search front and left side

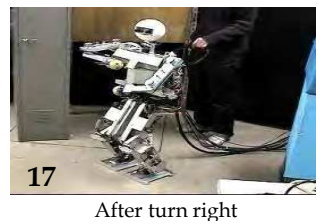

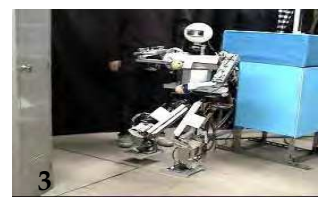

Side-step to right

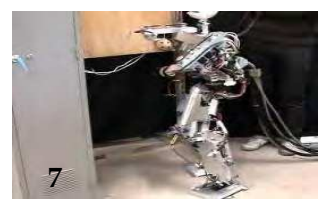

Correct angle left leg

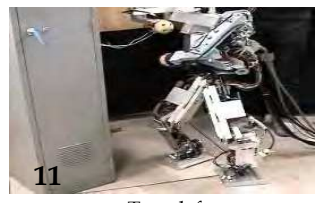

Turn left

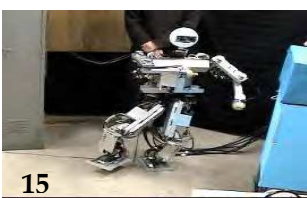

Right leg turn right

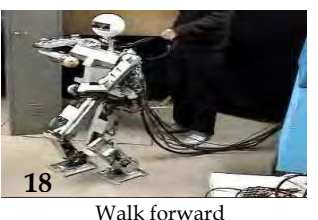

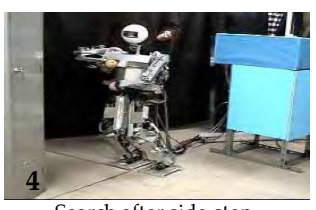

Search after side-step

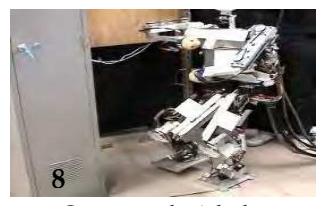

Correct angle right leg
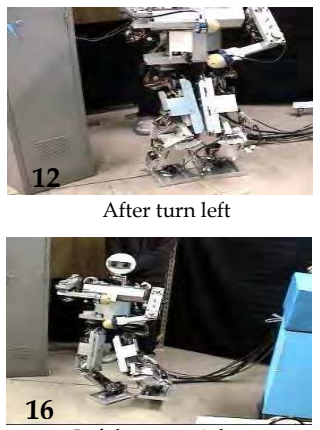

Left leg turn right

Fig. 25. Sequential photographs of humanoid robot locomotion in navigation experiment. The visual sensors were not connected to the system; therefore the robot locomotion had to rely on the contact interaction of the arms equipped with force sensors. 


\section{Conclusion}

Research on humanoid robots in areas related with human-robot interaction has rapidly increased recently especially for application to human living environments and emergency sites. It is apparent that the environments to be shared by humanoid robots are normally dedicated to humans. In this chapter we presented analysis results of optimum biped trajectory planning for humanoid robot navigation to minimize possibility of collision during operation in unseen environment. In this analysis, we utilized 21-dof biped humanoid robot Bonten-Maru II. In this chapter, at first we analyzed the joint trajectory generation in humanoid robot legs to define efficient gait pattern. We present kinematical solutions and optimum gait trajectory patterns for humanoid robot legs. Next, we performed analysis to define efficient walking gait locomotion by improvement of walking speed and travel distance without reducing reduction-ratio at joint-motor system. Next present analyses of collision checking using the robot arms to perform searching, touching and grasping motions in order to recognize its surrounding condition.

The presented biped trajectory analysis and planning improved performance of the navigation system. This was proved by simulation and experimental results applying biped humanoid robot Bonten-Maru II during performing biped walk, side-step and yawing motions. Furthermore, analysis of efficient gait trajectory and pattern was presented in this chapter. The analysis results of the gait trajectory generation proposed an efficient gait pattern for the biped robot. Meanwhile, regarding to speed-up walk analysis, simulation results based on humanoid robot Bonten-Maru II parameters revealed that walking speed was improved by applying low duty-ratio at suitable step length and hip-joint height, whereby the walking speed increased about two times compared to normal condition. Moreover, real-time experiments utilizing real biped humanoid robot based on the simulation results showed that the robot's travel distance during walking was improved about three times longer than current walking condition. This analysis results proved that it is possible to improve walking speed in a stable biped locomotion without reducing the reduction-ratio in the robot joint-motor system. Consequently the high torque output at the robot's manipulator to conduct tasks in various motions is maintained.

We have presented collision checking method in conjunction with the motion algorithm in the contact sensing-based navigation system. The collision checking is defined by searching motions of the robot's arms that created a radius of detection area within the arm's reach, which is treated as collision free area when no object is detected. Consequently the robot control system created an absolute collision free area for the robot to safely generate trajectories in the navigation tasks. When object is detected during searching motion, the robot arm will touch and grasp the object surface to define self-localization. At this moment, the navigation map is refined and a new path is planned automatically.

The above analysis results contribute to the effort to create a stable and reliable biped walking locomotion during performing tasks in the proposed navigation system. Finally, the proposed algorithm was evaluated in an experiment with a prototype humanoid robot operating in a room with walls and obstacles. The experimental results revealed good performance of the robot locomotion in recognizing the environmental conditions and generating suitable locomotion to walk safely towards the target point. Finally, the proposed strategy was demonstrated to have good potential to support current visual-based navigation systems so that humanoids can further 'adapt' in real environment. 


\section{References}

Chevallereau, C. \& Aoustin, Y. (2001). Optimal trajectories for walking and running of a biped robot, Robotica, Vol. 19, Issue 5, pp. 557-569

Denavit, J. \& Hartenberg, S. (1995). A kinematics notation for lower-pair mechanisms based upon matrices, Journal of Applied Mechanics, Vol. 77, pp. 215-221

Diaz, J. F.; Stoytchev, A. \& Arkin, R. C. (2001). Exploring unknown structured environments, Proceeding 14th Int. Florida Artificial Intelligent Research Society Conference (FLAIRS01), pp. 145-149

Dillmann, R. (2004). Teaching and learning of robot tasks via observation of human performance, Journal Robotics and Autonomous Systems, Vol. 47, Issue2-3, pp. 109-116

Gutmann, J.; Fukuchi, M. \& Fujita, M. (2005). Real-time path planning for humanoid robot navigation, Proceeding of the Int. Joint Conference on Artificial Intelligent, pp. 1232-1238

Hanafiah Y.; Ohka, M.; Yamano, M. \& Nasu, Y. (2008). Navigation strategy by contact sensing interaction for a biped humanoid robot, International Journal of Advanced Robotic Systems (ARS), Vol. 5, No. 2, June 2008, pp. 151-160, ISSN 1729-8806

Hanafiah Y.; Yamano, M.; Ohka, M. \& Nasu, Y. (2007). Development of a contact interactionbased navigation strategy for a biped humanoid robot, Proceedings of 2007 IEEE/RSJ International Conference on Intelligent Robots and Systems (IROS'07), pp. 4241-4246, San Diego, California, USA

Hirai, K.; Hirose, M.; Haikawa, Y. \& Takenaka, T. (1998). The development of Honda humanoid robot, Proceedings of International Conference on Robotics and Automation'98, pp. 1321-1326

Hirukawa, H.; Kanehiro, F. \& Kaneko, K. (2004). Humanoid robotics platforms developed in HRP, Journal of Robotics and Automation Systems, Vol. 48, pp. 165-175

Ishiguro, H. (2007). Scientific issues concerning androids, International Journal of Robotic Research, Vol. 26, pp. 105-117

Kajita, S.; Nagasaki, T.; Kaneko, K.; Yokoi, K. \& Tanie, K. (2005). A running controller of humanoid biped HRP-2LR, Proceeding of the 2005 IEEE International Conference on Robotics and Automation (ICRA2005), pp. 618-624, Barcelona, Spain

Khatib, O.; Yokoi, K. \& Casal, A. (1999). Robots in human environments: Basic autonomous capabilities, Journal of Robotics Research, Vol. 18, No. 7, pp. 684-696

Kuffner, J.; Nishikawa, K.; Kagami, S.; Kuniyoshi, Y.; Inaba, M. \& Inoue, H. (2002). Selfcollision detection and prevention for humanoid robots, Proceeding of 2002 IEEE International Conference on Robotics and Automation (ICRA2002), Vol. 3, pp. 2265-2270

Liu, H.; Zha, H.; Chen, K. \& Wang P. (2002). A new real-time collision prediction model for soccer robots, Proceeding Korea-China Joint Workshop on Intelligent Systems, pp.54-59, Seoul, Korea

Ogata, T.; Sugano, S. \& Tani, J. (2005). Acquisition of motion primitives of robot in humannavigation task: Towards human-robot interaction based on "Quasi-Symbol", Journal of Japanese Society for Artificial Intelligence, Vol.20, No.3, pp.188-196

Ogura, Y.; Ando, S.; Lim, H. \& Takanishi, A. (2004). Sensory-based walking motion instruction for biped humanoid robot, Journal Robotics and Autonomous Systems, Vol. 48 , pp. $223-230$

Okada, K.; Inaba, M. \& Inoue, H. (2003). Integration of real-time binocular stereo vision and whole body information for dynamic walking navigation of humanoid robot, Int. Conf. on Multisensor Fusion and Integration for Intelligent Systems, pp. 131-136 
Sagues, C. \& Guerrero, J. J. (1999). Motion and structure for vision-based navigation, Journal Robotica, Vol. 17, pp. 355-364

Salter, T.; Dautenhahn, K. \& Boekhorst R. (2006). Learning about natural human-robot interaction styles, Journal of Robotics and Autonomous Systems, Vol. 52, Issue 2, pp.127-134

Seara, J.F. \& Schmidt, G. (2004). Intelligent gaze control for vision-guided humanoid walking: methodological aspects, Journal of Robotics and Autonomous System, Vol. 48, pp. 231-248

Stasse, O.; Davison, J.A.; Sellaouti, R. \& Yokoi, K. (2006). Real-time 3D SLAM for humanoid robot considering pattern generator information, Proceeding IEEE/RSJ International Conference on Intelligent Robots and Systems 2006 (IROS2006), pp. 348-355, China

Perry, J. (1992). Gait analysis: Normal and pathological function. Slack Incorporated, pp. 4-6

Thompson, S.; Kida, Y.; Miyazaki, A. \& Kagami, S. (2006). Real time autonomous navigation with a 3D laser range sensor, Proceeding $3^{\text {rd }}$ Int. Conf. on Autonomous Robot and Agents (ICARA06), pp. 1-8, December 2006, Palmerston North, New Zealand

Yamaguchi, J.; Takanishi, A. \& Kato, I. (1993). Development of a biped walking robot compensating for three-axis moment by trunk motion, Proceeding of 2003 EEE/RSJ International Conference on Intelligent Robots and Systems (IROS '93)

\section{Appendix}

$\begin{array}{lll}\sum_{1} & : & \text { Joint-coordinate frame } \\ \mathbf{T} & : \text { Homogeneous transformation matrix } \\ \text { Rot } & : \text { Rotation transformation } \\ \text { Trans } & : \text { Transfer/offset transformation } \\ \theta_{i} & : \text { Joint angle between the } \mathrm{X}_{i-1} \text { and the } \mathrm{X}_{i} \text { axes measured about the } \mathrm{Z}_{i} \text { axis } \\ d_{i} & : \text { Distance from the } \mathrm{X}_{i-1} \text { axis to the } \mathrm{X}_{i} \text { axis measured along the } \mathrm{Z}_{i} \text { axis } \\ \alpha_{i} & : \text { Angle between the } \mathrm{Z}_{i} \text { axis to the } \mathrm{Z}_{i-1} \text { axis measured about the } \mathrm{X}_{i-1} \text { axis } \\ l_{i} & : \text { Distance from the } \mathrm{Z}_{i} \text { axis to the } \mathrm{Z}_{i-1} \text { axis measured along the } \mathrm{X}_{i-1} \text { axis } \\ s_{i j} & : \text { Sin }\left(\theta_{i}+\theta_{j}\right) \\ c_{i j} & : \text { Cos }\left(\theta_{i}+\theta_{j}\right) \\ \text { atan2 } & : \text { Arc tangent of two variables } \\ { }_{h} \mathbf{R} & : \text { Matrix describes end-effector orientation with respect to reference coordinate } \\ { } \mathbf{P}_{h} & : \text { Matrix describes end-effector position with respect to reference coordinate } \\ P_{x, y, z} & : \text { Position of end-effector at } \mathrm{x}, \mathrm{y}, \mathrm{Z} \text { axes } \\ P_{0} & : \text { End-effector starting position } \\ P_{f} & : \text { End-effector finished position } \\ u & : \text { Ratio of current time and motion time }\end{array}$




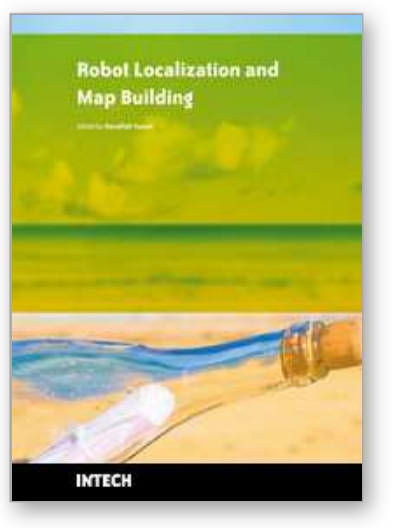

\author{
Robot Localization and Map Building \\ Edited by Hanafiah Yussof
}

ISBN 978-953-7619-83-1

Hard cover, 578 pages

Publisher InTech

Published online 01, March, 2010

Published in print edition March, 2010

Localization and mapping are the essence of successful navigation in mobile platform technology. Localization is a fundamental task in order to achieve high levels of autonomy in robot navigation and robustness in vehicle positioning. Robot localization and mapping is commonly related to cartography, combining science, technique and computation to build a trajectory map that reality can be modelled in ways that communicate spatial information effectively. This book describes comprehensive introduction, theories and applications related to localization, positioning and map building in mobile robot and autonomous vehicle platforms. It is organized in twenty seven chapters. Each chapter is rich with different degrees of details and approaches, supported by unique and actual resources that make it possible for readers to explore and learn the up to date knowledge in robot navigation technology. Understanding the theory and principles described in this book requires a multidisciplinary background of robotics, nonlinear system, sensor network, network engineering, computer science, physics, etc.

\title{
How to reference
}

In order to correctly reference this scholarly work, feel free to copy and paste the following:

Hanafiah Yussof and Masahiro Ohka (2010). Optimum Biped Trajectory Planning for Humanoid Robot Navigation in Unseen Environment, Robot Localization and Map Building, Hanafiah Yussof (Ed.), ISBN: 978953-7619-83-1, InTech, Available from: http://www.intechopen.com/books/robot-localization-and-mapbuilding/optimum-biped-trajectory-planning-for-humanoid-robot-navigation-in-unseen-environment

\section{INTECH}

open science | open minds

\section{InTech Europe}

University Campus STeP Ri

Slavka Krautzeka 83/A

51000 Rijeka, Croatia

Phone: +385 (51) 770447

Fax: +385 (51) 686166

www.intechopen.com

\section{InTech China}

Unit 405, Office Block, Hotel Equatorial Shanghai

No.65, Yan An Road (West), Shanghai, 200040, China

中国上海市延安西路65号上海国际贵都大饭店办公楼 405 单元

Phone: +86-21-62489820

Fax: $+86-21-62489821$ 
(C) 2010 The Author(s). Licensee IntechOpen. This chapter is distributed under the terms of the Creative Commons Attribution-NonCommercialShareAlike-3.0 License, which permits use, distribution and reproduction for non-commercial purposes, provided the original is properly cited and derivative works building on this content are distributed under the same license. 